\title{
Chronic senescent human mesenchymal stem cells as possible contributor to the wound healing disorder after exposure to the alkylating agent sulfur mustard
}

\author{
Simone Rothmiller ${ }^{1,2} \cdot$ Niklas Jäger $^{1} \cdot$ Nicole Meier $^{1} \cdot$ Thimo Meyer $^{1} \cdot$ Adrian Neu $^{1} \cdot$ Dirk Steinritz $^{1,3}$. \\ Horst Thiermann ${ }^{1} \cdot$ Michael Scherer $^{4} \cdot$ Christoph Rummel $^{5}$ - Aswin Mangerich ${ }^{2} \cdot$ Alexander Bürkle $^{2}$. \\ Annette Schmidt ${ }^{1,6}$ (i)
}

Received: 27 July 2020 / Accepted: 28 October 2020 / Published online: 25 January 2021

(C) The Author(s) 2021

\begin{abstract}
Wound healing is a complex process, and disturbance of even a single mechanism can result in chronic ulcers developing after exposure to the alkylating agent sulfur mustard (SM). A possible contributor may be SM-induced chronic senescent mesenchymal stem cells (MSCs), unable to fulfil their regenerative role, by persisting over long time periods and creating a proinflammatory microenvironment. Here we show that senescence induction in human bone marrow derived MSCs was time- and concentration-dependent, and chronic senescence could be verified 3 weeks after exposure to between 10 and $40 \mu \mathrm{M}$ SM. Morphological changes, reduced clonogenic and migration potential, longer scratch closure times, differences in senescence, motility and DNA damage response associated genes as well as increased levels of proinflammatory cytokines were revealed. Selective removal of these cells by senolytic drugs, in which ABT-263 showed initial potential in vitro, opens the possibility for an innovative treatment strategy for chronic wounds, but also tumors and age-related diseases.
\end{abstract}

Keywords Senescence $\cdot$ Mesenchymal stem cells - Wound healing disorder $\cdot$ Chemical warfare agents $\cdot$ Sulfur mustard

\section{Introduction}

Supplementary Information The online version contains supplementary material available at https://doi.org/10.1007/s0020 4-020-02946-5.

Annette Schmidt

annette.schmidt@unibw.de

1 Bundeswehr Institute of Pharmacology and Toxicology, Neuherbergstraße 11, 80937 Munich, Germany

2 Molecular Toxicology Group, Department of Biology, University of Konstanz, 78457 Konstanz, Germany

3 Walther-Straub-Institute of Pharmacology and Toxicology, University of Munich, Goethestr. 33, 80336 Munich, Germany

4 Department of Traumatology and Orthopedics, HELIOS Amper Clinics, Krankenhausstraße 15, 85221 Dachau, Germany

5 Department of Orthopedics and Sports Medicine, Wolfart Clinic, Waldstraße 7, 82166 Gräfelfing, Germany

6 Faculty of Human Sciences, Institute for Sports Sciences, Universität Der Bundeswehr München, Werner-Heisenberg-Weg 39, 85577 Neubiberg, Germany
Damages in the natural barrier of the skin against the environment set the complex wound healing process into motion, during which the skin and underlying tissue repair themselves (Bukowiecki et al. 2017). Mesenchymal stem cells (MSCs) are multipotent adult stem cells and play an essential role in wound healing by accelerating wound closure, enhancing re-epithelialization, increasing angiogenesis, promoting granulation tissue formation, modulating inflammation, and regulating extracellular matrix remodeling (Lee et al. 2016). MSCs home to damaged tissue, and upon arrival, they exert their therapeutic effects mainly by paracrine signaling via various cytokines, immunosuppressive, growth and differentiation factors (Ranganath et al. 2012; Ma et al. 2014). Because of their self-renewal potential, simple isolation process and expansion in vitro, MSCs are a promising tool in regenerative medicine (Bruder et al. 1998) and multiple studies already showed that MSCs enhance wound healing by accelerating wound closure (Walter et al. 2010; Nie et al. 2011; Rodriguez-Menocal et al. 2015). 
a

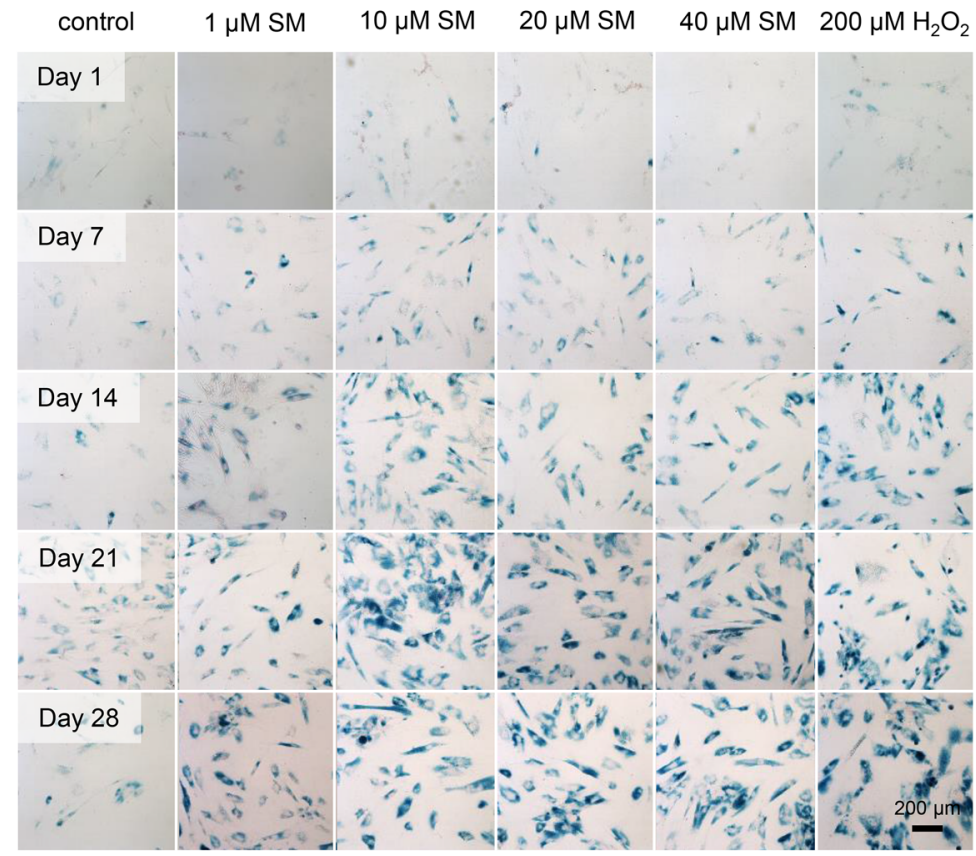

b

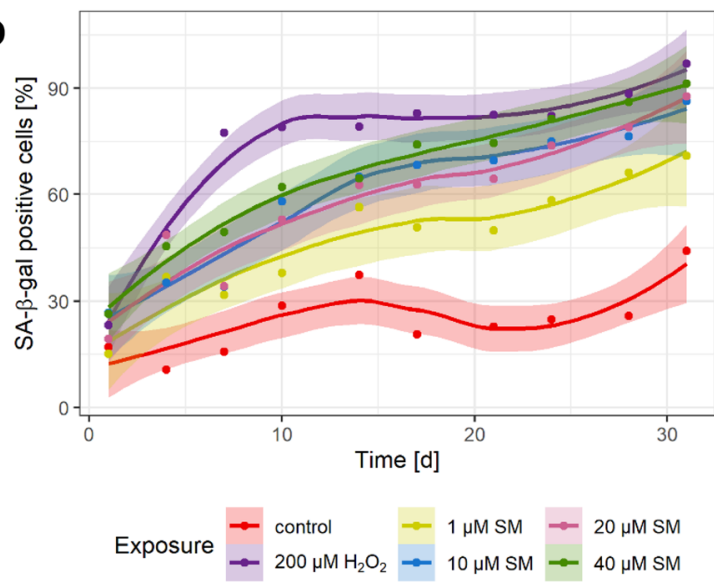

C

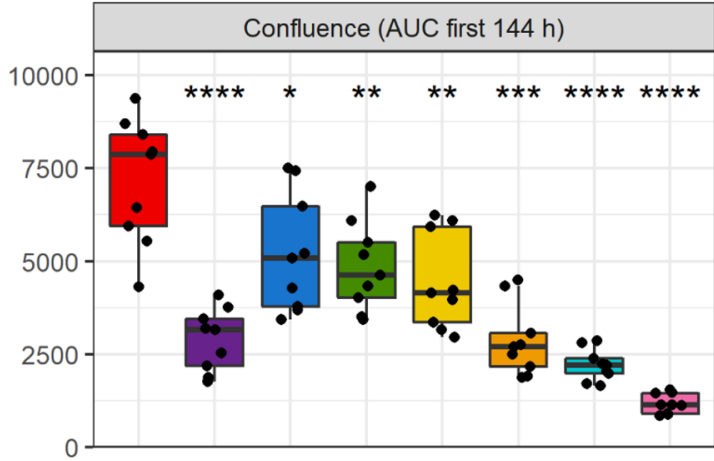

d

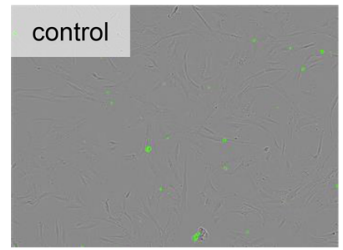

\section{$40 \mu \mathrm{M} \mathrm{SM}$}
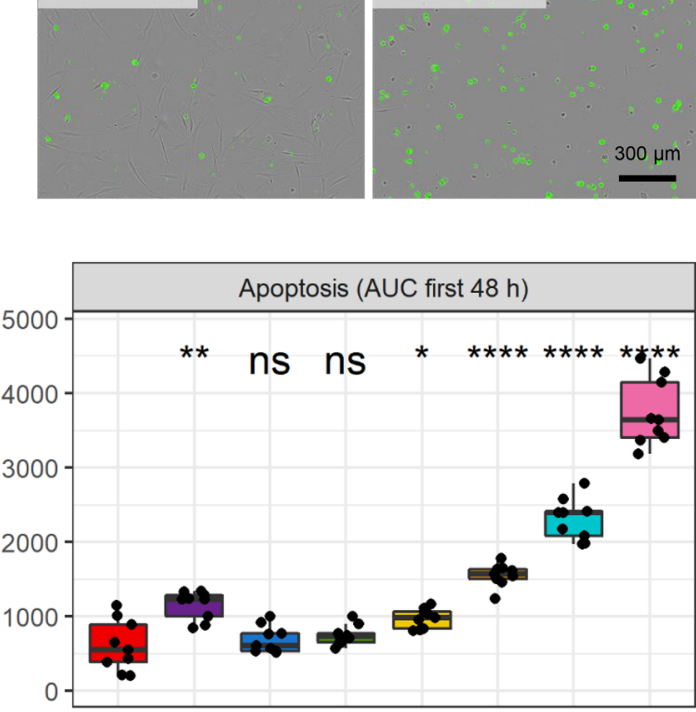

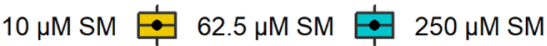

$40 \mu \mathrm{M} \mathrm{SM}$
Failure in wound healing or some diseases, e.g., diabetes mellitus, lead to chronic wounds, that not only reduce the patients' quality of life but also create a significant financial burden on the healthcare system (Augustin and
Maier 2003). Chronic wounds have also been described after exposure to the alkylating chemical warfare agent sulfur mustard (SM) (Schmidt et al. 2013). Although SM has been banned by the Chemical Weapons Convention, it 
४Fig. 1 Senescence and apoptosis induction. a Representative images of concentration- and time-dependent development of SA- $\beta$-gal staining (blue) after single dose $\mathrm{SM}$ or $\mathrm{H}_{2} \mathrm{O}_{2}$ exposure (day 0) in contrast to solvent controls. Counterstaining of all cells with nuclear fast red (red). Scale bar, $200 \mu \mathrm{m}$. b The means with a trend including $99 \%$ confidence intervals of the percentage of SA- $\beta$-gal positive cells counted every 3-4 days over 31 days $(n=$ three randomly selected fields per group from three independent experiments). c Area under the curve (AUC) for confluence (area covered by cells) within the first $144 \mathrm{~h}$ and apoptosis (d annexin V positive cells (green), representative images $t=48 \mathrm{~h}$ ) within the first $48 \mathrm{~h}$. Shown is the significance between solvent controls and $200 \mu \mathrm{M} \mathrm{H}_{2} \mathrm{O}_{2}$ or SM exposed MSCs ( $\mathrm{n}=$ three randomly selected images per group from three independent experiments). Data are represented as Tukey boxplots; $* p<0.05$, $* * p<0.01, * * * p<0.001, * * * * p<0.0001$. See also Supplementary Figs. 1-3

was most recently used in Syria (Kilic et al. 2018; Sezigen et al. 2019; John et al. 2019). Mainly the easy synthesis and stockpiles still existing in various countries enable acute threats of terroristic attacks, which highlights the importance of developing countermeasures. Despite intensive research, the molecular toxicity of SM is incompletely understood, there is no antidote or prophylaxis available and the treatment is only symptomatic (Etemad et al. 2019). Skin is one of the main target organs of SM and skin lesions occurred in more than $90 \%$ of exposed persons (Emadi et al. 2008). In detail, after a characteristic latency period without any clinical presentations, SM causes edema, inflammation, skin blisters and ulceration. Skin lesions may take months to heal and often result in chronic wounds, which may require skin grafting (Schmidt et al. 2018b). Additionally to problematic skin damages after primary healed SM affections, it was discovered during World War I that sites of the body with previous SM exposure which had already partially or entirely healed, became active again in some patients when the same individual was exposed to SM even years later at other, distant skin sites ("flare-ups") (Sulzberger et al. 1947).

Because MSCs show important properties in wound healing, they may be affected during SM poisoning. This is underlined by the fact that MSCs derived from patients suffering from chronic wound healing disorders showed reduced migratory attraction to skin and wound fibroblasts compared to healthy donors (Rodriguez-Menocal et al. 2012). SM might be able to affect MSCs in bone marrow, as it was shown that SM victims demonstrated bone marrow depletion similar to cytostatic treatment (Hassan and Ebtekar 2002). Indeed, bone marrow identified as was one of the most sensitive tissues in terms of DNA crosslinks (Yue et al. 2015). We could already demonstrate that although MSCs are highly resistant against SM in terms of cell survival, even low doses do reduce the migration (Schmidt et al. 2013) and proliferation but not apoptosis (Schmidt et al. 2018a). We could also show dramatic changes in the secretome and increased migration by addition of specific cytokines (Schreier et al. 2018).

Chronic wounds and flare-ups are long-term complications after SM exposure and thus there needs to be some kind of "memory", which may be senescent cells due to their longevity. Senescence is part of many cellular mechanisms including aging (Baker et al. 2016), age-related diseases (Baker et al. 2011), tissue remodeling (Krizhanovsky et al. 2008), wound healing (Jun and Lau 2010) and immunity (Kearney et al. 2015). Recently, senescence was defined as a specific permanent growth arrest of proliferation-competent cell induced by stressors (Sharpless and Sherr 2015) like reactive oxygen species (ROS) (von Zglinicki 2002) or unresolved DNA damage (Sedelnikova et al. 2004). Most routinely the expression of senescence-associated $\beta$-galactosidase (SA- $\beta$-gal), p16 ${ }^{\mathrm{INK} 4 \mathrm{~A}}$ and the senescenceassociated secretory phenotype (SASP) are used as biomarkers. Affected cells are not eliminated by the immune system, persist over months or years and secrete proinflammatory factors (Sharpless and Sherr 2015). Thus, senescent MSCs may play a role in the chronic wound healing disorder after SM exposure. A treatment using senolytic drugs, i.e., natural products or small synthetic molecules that selectively eliminate senescent cells, as it has already been successfully tested in human MSCs after replicative senescence (Grezella et al. 2018), could clear senescent MSCs after SM exposure. In this study, we investigated whether SM could induce senescence in MSCs and thereby may influence wound healing as well as whether senolytic drugs are a promising option for treatment of SM induced wounds.

\section{Results}

\section{Senescence and apoptosis induction in mesenchymal stem cells by sulfur mustard and hydrogen peroxide}

For all experiments, human bone marrow derived MSCs were used (Supplementary Fig. 1a-h), and their identity was checked regularly by morphology, expression of cell surface markers and differentiation potential (Supplementary Fig. 1i, j). SM concentrations from $\mathrm{LC}_{1}(1 \mu \mathrm{M})$ to $\mathrm{LC}_{25}$ $(40 \mu \mathrm{M})($ Schmidt et al. 2013) were used initially to test whether a single dose exposure can induce senescence in MSCs. SA- $\beta$-gal staining was used to determine the percentage of senescent cells at different time points after exposure and $\mathrm{H}_{2} \mathrm{O}_{2}$ was used as a positive senescence induction control (Brandl et al. 2011). As shown in Supplementary Fig. 1k, cell survival after $\mathrm{H}_{2} \mathrm{O}_{2}$ exposure was determined at the half-maximal lethal concentration $\left(\mathrm{LC}_{50}\right)$ of $322.7 \pm 11.4 \mu \mathrm{M}$. Therefore, $200 \mu \mathrm{M} \mathrm{H}_{2} \mathrm{O}_{2}\left(\mathrm{LC}_{16}\right)$ was used for subsequent experiments. To assess the concentration and 

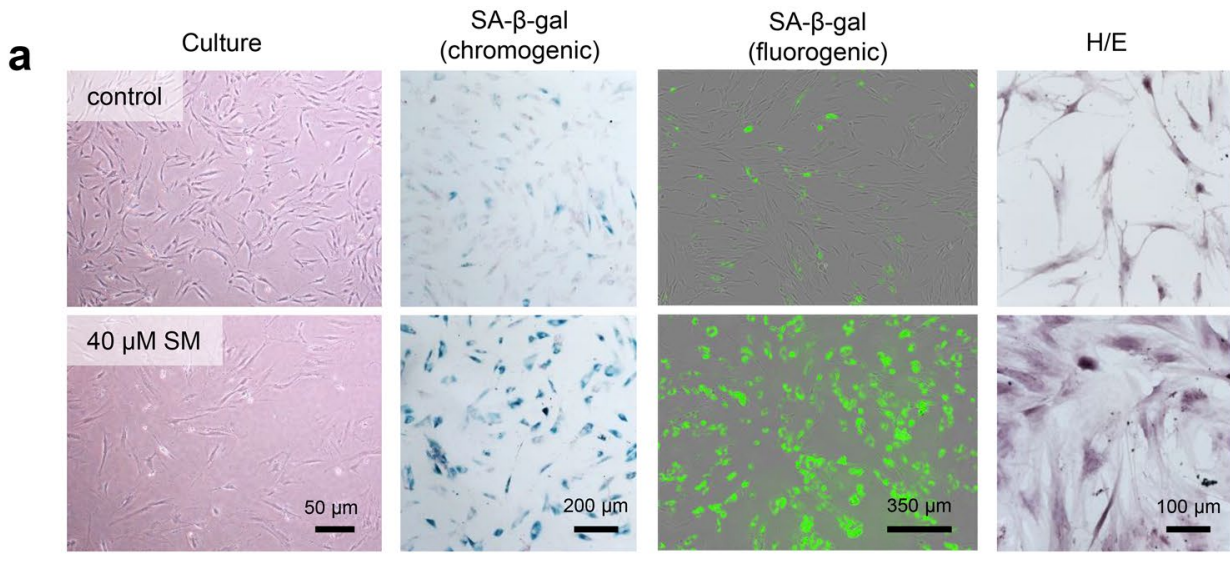

\section{b}

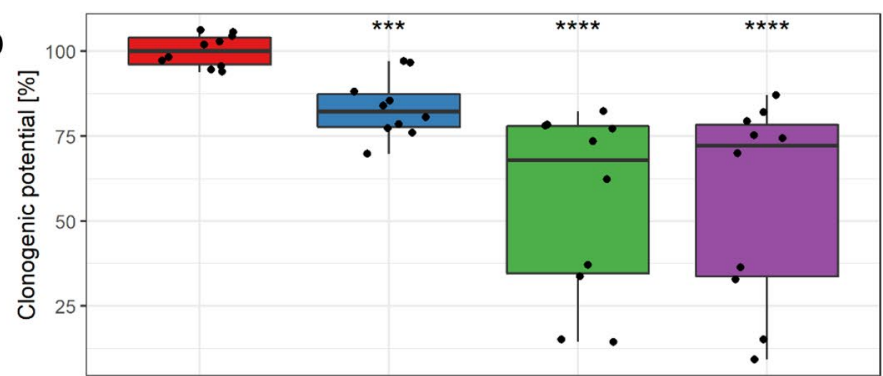

Cell type

\section{C}
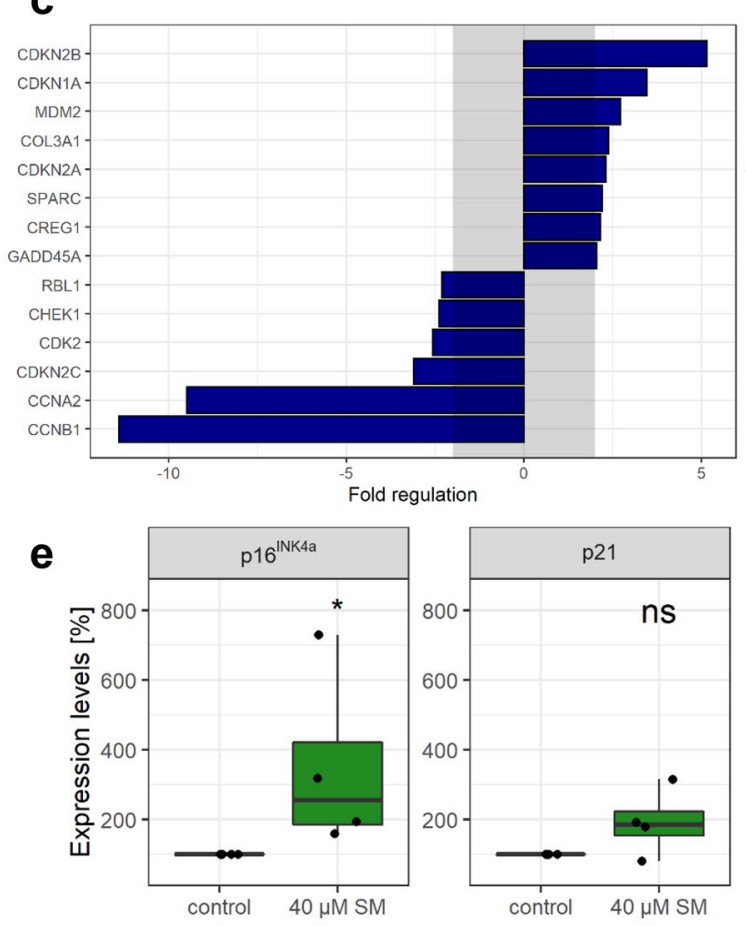

time dependence of senescence induction, staining was performed up to 31 day post-exposure (day 0), with intervals of 3-4 days. As is shown in Fig. 1a, b, the percentage of senescent cells as well as the staining intensity increased with time. In the concentration range from 10 to $40 \mu \mathrm{M}$ $\mathrm{SM}$, almost all cells were senescent within 21 days and no

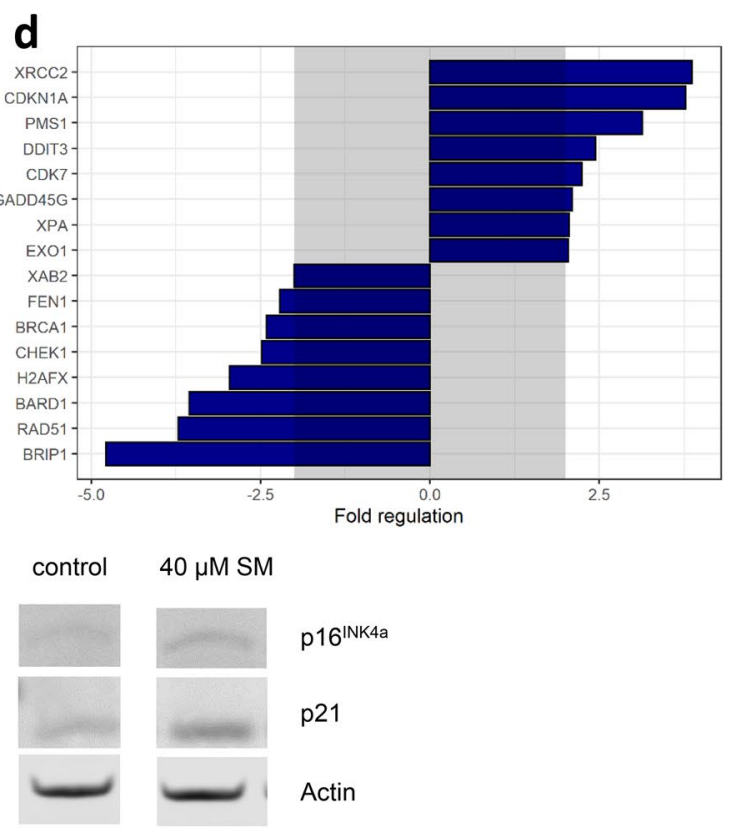

obvious change in senescent state was seen thereafter. The exposure to $200 \mu \mathrm{M} \mathrm{H}_{2} \mathrm{O}_{2}$ showed comparable results, but with a stronger initial increase. In comparison, $1 \mu \mathrm{M}$ SM did not lead to complete senescence. Increased percentages of SA- $\beta$-gal positive cells reached the significance level at days 7, 14, 21 and 28 post-exposure, when compared to solvent 
४Fig. 2 Senescence markers. a Representative images from both chromogenic (PromoCell) and fluorogenic (Cell Biolabs) SA- $\beta$-gal staining in MSCs 21 days after $40 \mu \mathrm{M} \mathrm{SM}$ exposure show a very high percentage of senescent cells with high staining intensity compared to solvent controls. Flattening and enlargement shown already in culture as well as by hematoxylin/eosin (H/E) staining. b Loss of replicative potential shown by clonogenicity assay. Colonies formed and stained with crystal violet could be observed for controls, but only single cells for $40 \mu \mathrm{M}$ SM senescent cells. After destaining with methanol, crystal violet absorbance examined at $570 \mathrm{~nm}$ by plate reader and mean of controls set to $100 \%$ ( $n=$ duplicates per group from five independent experiments). Genes associated with c senescence biomarkers or d DNA damage and DNA repair were tested 21 days after $40 \mu \mathrm{M}$ SM exposure and compared to controls. After RNA extraction from MSCs, a RT-qPCR assay was performed and fold regulation of up- or downregulated genes $(\geq 2.0$ or $\leq-2.0$ in combination with $p<0.05$, outside grey box) are shown as means ( $n=$ three independent experiments). e The upregulation of p16 $6^{\mathrm{INK} 4 \mathrm{a}}$ and $\mathrm{p} 21$ was also observed using Western Blot. Representative bands and expression levels are shown $(\mathrm{n}=$ four independent experiments). Data are represented as Tukey boxplots; ${ }^{*} p<0.05,{ }^{* *} p<0.01$, *** $p<0.001$, $* * * * p<0.0001$. See also Supplementary Fig. 4

controls (Supplementary Fig. 2a). Solvent controls also showed a slight increase in senescence over time, but the conditions were adjusted to keep senescence as low as possible, for example using low passage numbers. For subsequent experiments, cells 21 days after exposure to 10 and $40 \mu \mathrm{M}$ SM or $200 \mu \mathrm{M} \mathrm{H}_{2} \mathrm{O}_{2}$, respectively, were considered suitable to be used as senescent cell types and cells exclusively exposed to solvent as non-senescent controls, respectively.

Full replicative senescence occurred not before 13 passages in controls (Supplementary Fig. 3a). Exposure to $40 \mu \mathrm{M} \mathrm{SM}$ resulted in a chronic senescence, since no replication of those cells occurred up to 24 weeks after induction. In comparison, upon $10 \mu \mathrm{M} \mathrm{SM}$ or $200 \mu \mathrm{M} \mathrm{H}_{2} \mathrm{O}_{2}$ exposure of MSCs, some cells seemed to reenter the cell cycle, based on an increase in cell numbers up to 24 weeks afterwards, but all three exposures resulted still in many SA- $\beta$-gal positive cells (Supplementary Fig. 3b). As a proof of principle, we also used commercially available adipose tissue derived MSCs and exposed them to 10, 20 and $40 \mu \mathrm{M}$ SM or $200 \mu \mathrm{M}$ $\mathrm{H}_{2} \mathrm{O}_{2}$. After 21 days, many SA- $\beta$-gal positive cells could be observed in all groups compared to the solvent control (Supplementary Fig. 3c). Moreover, senescence could also be induced by continuous $\mathrm{SM}$ or $\mathrm{H}_{2} \mathrm{O}_{2}$ exposure. While $1 \mu \mathrm{M} \mathrm{SM}$ or $50 \mu \mathrm{M} \mathrm{H}_{2} \mathrm{O}_{2}$ two to three times per week was sufficient, 0.1 or $0.5 \mu \mathrm{M} \mathrm{SM}$ was too low to result in close to $100 \%$ SA- $\beta$-gal positive cells after 21 days (Supplementary Fig. $3 d$ ). The SA- $\beta$-gal positive senescent phenotype of MSCs 21 days after exposure to $40 \mu \mathrm{M}$ SM was not only comparable to $200 \mu \mathrm{M} \mathrm{H}_{2} \mathrm{O}_{2}$, but also to exposure with DNA-damaging chemotherapeutic agents like $20 \mu \mathrm{M}$ cisplatin, $50 \mu \mathrm{M}$ melphalan and $50 \mu \mathrm{M}$ bendamustine or ionizing radiation with 10 Gy (Supplementary Fig. 3e).

Since the concentrations used for senescence induction also reduced cell viability, the influence on cell growth and apoptosis was determined. After the exposure to increasing SM concentrations, $200 \mu \mathrm{M} \mathrm{H}_{2} \mathrm{O}_{2}$ or solvent, the confluence, i.e., area covered by cells, and apoptotic cells, i.e., number of cells positive for Annexin V, were recorded microscopically for more than 11 days. Supplementary Fig. 2b shows that the linear range of confluence increase in the solvent control was up to about $144 \mathrm{~h}$ and a maximum of apoptotic cells was reached within about $48 \mathrm{~h}$ determined by $500 \mu \mathrm{M}$ SM exposure. Following these observations, the area under the curve (AUC) was calculated within these time frames for statistical analysis. As shown in Fig. 1c, the confluence was significantly decreased when cells were exposed to $200 \mu \mathrm{M}$ $\mathrm{H}_{2} \mathrm{O}_{2}$ or $\mathrm{SM}$ in a concentration-dependent fashion from 10 to $500 \mu \mathrm{M}$, when compared to solvent controls. On the other hand, $200 \mu \mathrm{M} \mathrm{H}_{2} \mathrm{O}_{2}$ significantly increased apoptosis within $48 \mathrm{~h}$ post-exposure in comparison to controls. For SM exposure, 10 and $40 \mu \mathrm{M}$ did not result in a significant increase, while apoptosis was significantly higher for concentrations between 62.5 and $500 \mu \mathrm{M} \mathrm{SM}$ (Fig. 1c, d). Both confluence reduction and apoptosis increase were very similar from 10 to $62.5 \mu \mathrm{M} \mathrm{SM}$, and beyond $62.5 \mu \mathrm{M}$ they were concentration dependent. Interestingly, the SM concentrations 10 and $40 \mu \mathrm{M}$ resulted in senescence but not apoptosis.

\section{Senescence markers}

To verify that SM-exposed MSCs are senescent, a variety of methods and senescence biomarkers were used. In Fig. 2a, MSCs 21 days after exposure to $40 \mu \mathrm{M}$ SM showed increased SA- $\beta$-gal activity compared to solvent controls by the chromogenic substance X-Gal as well as by a fluorogenic substrate. Morphological changes like cell size increase and flattening could already be observed during culture, but also by H/E staining. The fluorogenic substrate was also used to identify SA- $\beta$-gal positive cells by flow cytometry and Supplementary Fig. 4a shows that exposure to 10 and $40 \mu \mathrm{M} \mathrm{SM}$ as well as $200 \mu \mathrm{M} \mathrm{H}_{2} \mathrm{O}_{2}$ increased the SA- $\beta$-gal positive cell population from $4.0 \%$ in solvent control to $46.7-58.8 \%$ after 3 weeks. Photomicrographs (Supplementary Fig. 4b) showed the increased cell size and granularity of the senescent cells in accordance with higher cell areas and side scatter intensity numbers (Supplementary Fig. 4c). Loss of proliferation of senescent cells could be shown as they had a significantly reduced colony forming ("clonogenic") potential. Non-senescent controls showed many grown colonies with more than 60 cells, while senescent cells were still mostly single cells only (Fig. $2 \mathrm{~b}$ ).

The expression levels of various senescence marker genes were observed to be significantly different (fold regulation $>2$ or $<-2$ in combination with $p<0.05)$ in senescent cells ( $40 \mu \mathrm{M}$ SM 21 days post-exposure) compared to non-senescent controls (Fig. 2c, Supplementary Table 1). Many genes involved in cell cycle control 

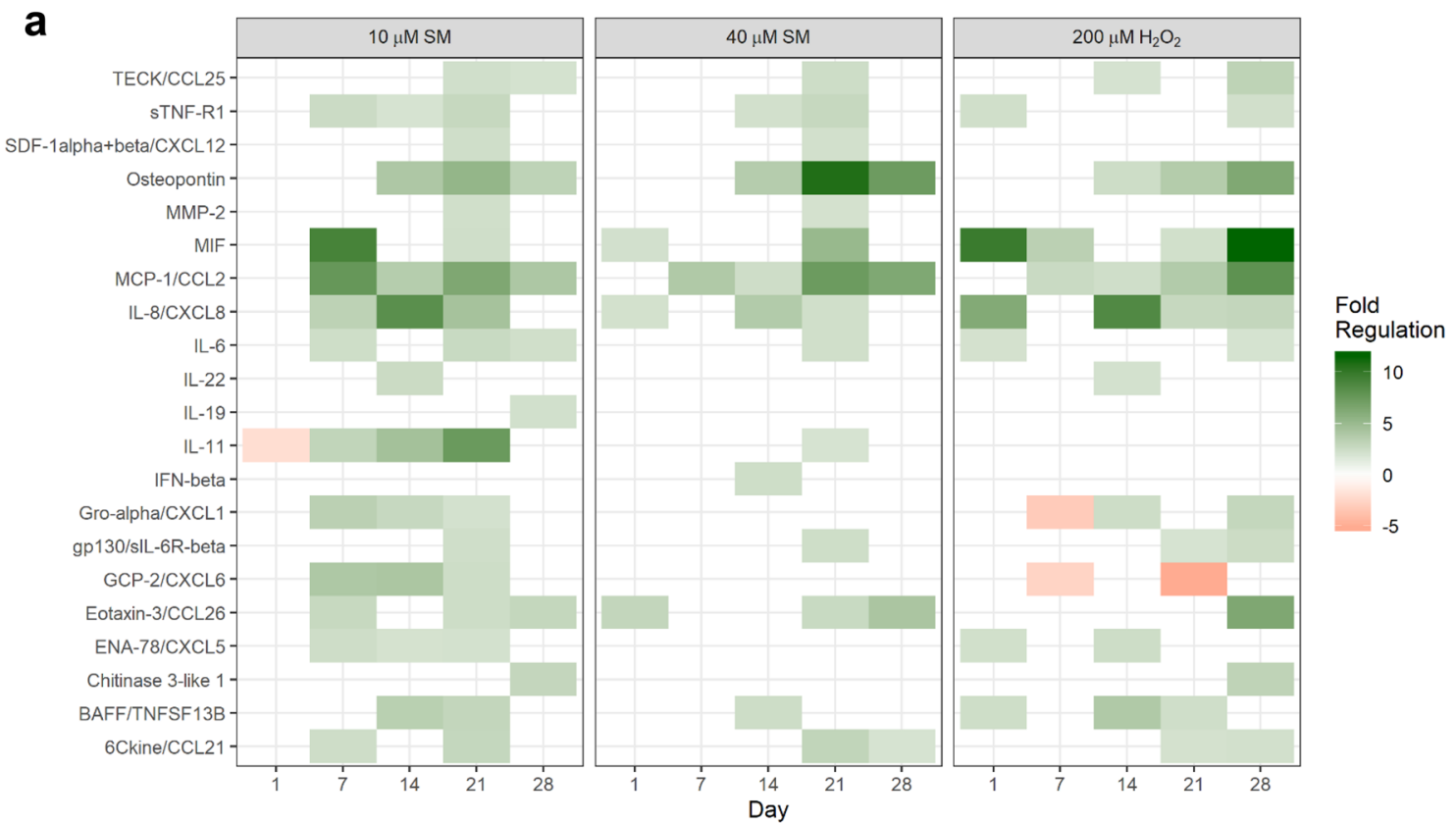

b
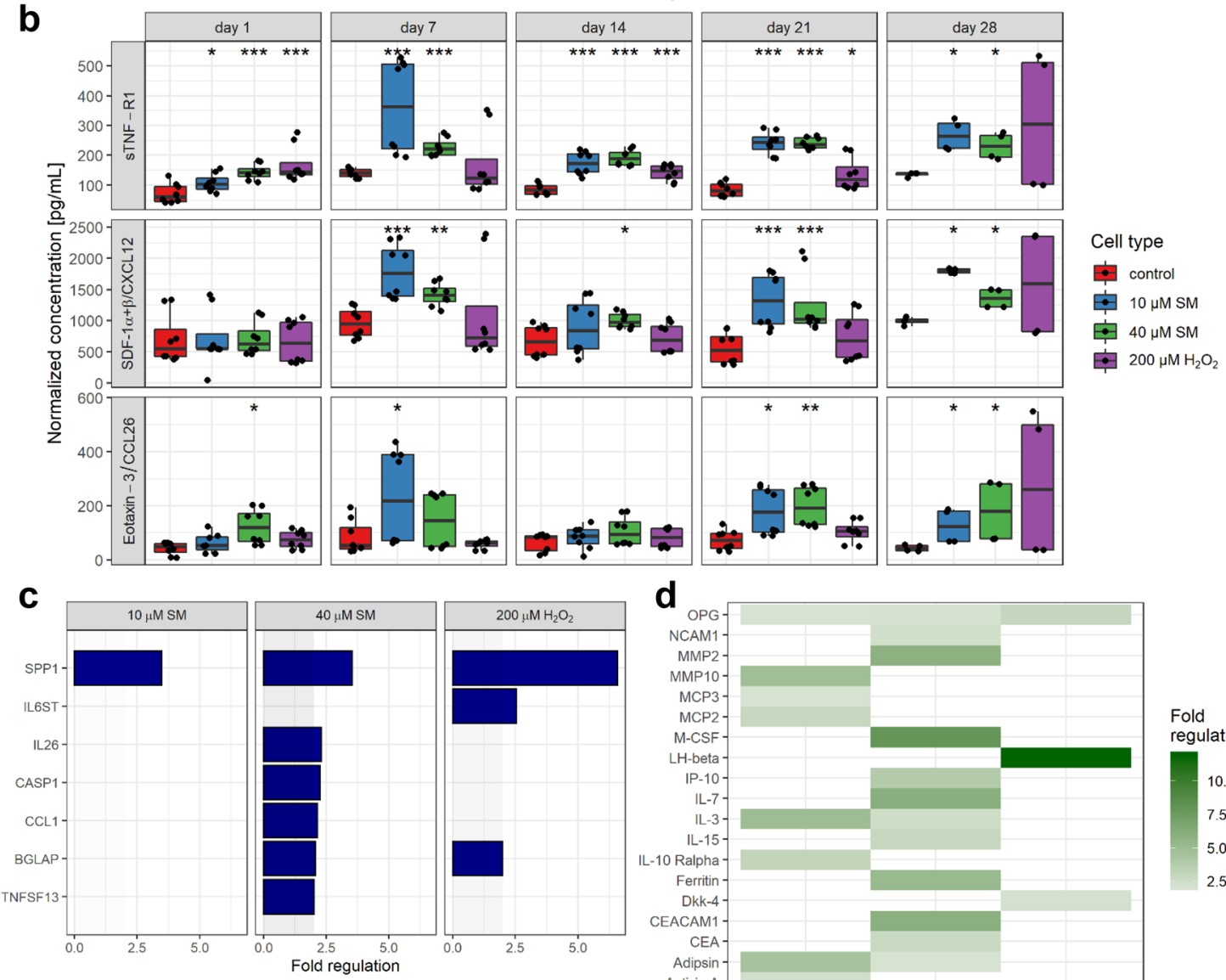

d

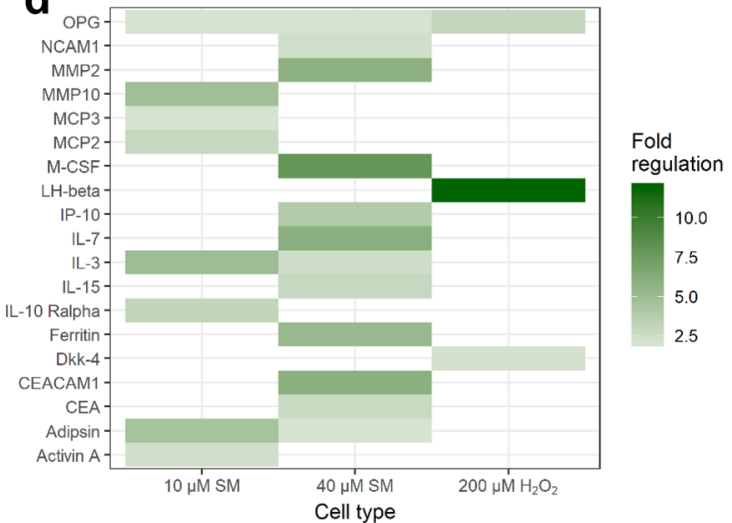

like 'cyclin-dependent kinase inhibitor 2B (CDKN2B)', The 'mouse double minute 2 homolog (MDM2)'; 'cel1A (CDKN1A, p21) and 2A (CDKN2A, p16 $\left.{ }^{\text {INK4a }}\right)$ were lular repressor of E1A-stimulated genes 1 (CREG1)'; more than 5-, 3- or twofold upregulated, respectively. 'collagen, type III, alpha 1 (COL3A1)'; 'secreted protein, 
4Fig. 3 Senescence-associated increase in proinflammatory factors. a, b The concentration of over 70 chemokines, cytokines and growth factors was determined by Bio-Plex assays in cell culture supernatants and normalized for the cell number $(n=$ biological duplicates per group from three independent experiments). a Fold regulation of up- or downregulated factors $(\geq 2.0$ or $\leq-2.0$ in combination with normalized concentrations above $20 \mathrm{pg} / \mathrm{mL}$ ) of senescent to nonsenescent controls are shown. b Three factors showed an upregulation in SM- but not in $\mathrm{H}_{2} \mathrm{O}_{2}$-exposed MSCs. c After RNA extraction from MSCs at day 21 after exposure to 10 and $40 \mu \mathrm{M} \mathrm{SM}, 200 \mu \mathrm{M}$ $\mathrm{H}_{2} \mathrm{O}_{2}$ or solvent, a RT-qPCR assay of a custom designed cytokine and chemokine gene panel was performed and fold regulation of up- or downregulated genes $(\geq 2.0$ or $\leq-2.0$ in combination with $p<0.05$, outside grey box) are shown as means $(n=$ three independent experiments). d Cell culture supernatants were collected 21 day post-exposure and freshly transferred to glass slide based ELISA cytokine arrays. Images were recorded with Odyssey scanner and spot intensities of 279 different factors calculated. After normalization with the positive controls, fold regulation was calculated for each senescent cell type against control and fold regulation of up- or downregulated factors are shown $(\geq 2.0$ or $\leq-2.0$ in combination with normalized concentrations above 5\%; $n=$ biological duplicates per group from two independent experiments). Data are represented as Tukey boxplots; $* p<0.05, * * p<0.01, * * * p<0.001$. See also Supplementary Fig. 5

acidic, cysteine-rich (SPARC)' and 'growth arrest and DNA-damage-inducible, $\alpha$ (GADD45A)' were also more than twofold upregulated. Down-regulated genes also included a variety of cell cycle regulators including 'retinoblastoma-like protein 1 (RBL1)'; 'checkpoint kinase 1 (CHEK1)'; 'cyclin-dependent kinase 2 (CDK2)'; 'cyclin-dependent kinase inhibitor 2C (CDKN2C)'; 'cyclin A2 (CCNA2)' and 'cyclin B1 (CCNB1)' between 2- and 11-fold downregulation.

Furthermore, the influence of SM-induced senescence on gene expression of DNA damage or repair related genes for persistently upregulated DNA damage response (DDR), another biomarker, was investigated (Fig. 2d, Supplementary Table 1). In senescent cells, besides CDKN1A between 2and almost fourfold upregulated expression was recorded for 'DNA-damage-inducible transcript 3 (DDIT3)'; 'growth arrest and DNA-damage-inducible, $\gamma$ (GADD45G)'; 'X-ray repair complementing defective repair in Chinese hamster cells 2 (XRCC2)'; 'exonuclease 1 (EXO1)'; 'postmeiotic segregation increased 1 (PMS1)'; 'Xeroderma pigmentosum, complementation group A (XPA)' and 'cyclin-dependent kinase 7 (CDK7)'. Interestingly, downregulated genes (by between 2- and almost fivefold) were almost all involved in the repair of DNA double strand breaks. Amongst these, 'breast cancer 1 , early onset (BRCA1)'; 'BRCA1 associated RING domain 1 (BARD1)'; 'BRCA1 interacting protein C-terminal helicase 1 (BRIP1)'; 'H2A histone family, member X (H2AFX)' and RAD51. Down-regulated genes were also found for other DNA repair mechanisms, besides CHEK1 the 'XPA binding protein 2 (XAB2)' and 'flap structure-specific endonuclease 1 (FEN1)'. No differences in gene expression were observed for poly (ADP-ribose) polymerases 1-3 (PARPs 1-3).

The upregulation of $\mathrm{p} 16^{\mathrm{INK} 4 \mathrm{a}}$ at the gene level corresponds with a significant increase in protein expression determined by western blot. The increase of p21 was significant on the gene, but not on the protein level (Fig. 2e).

\section{Increase in secreted proinflammatory factors due to senescence}

One very important aspect and biomarker of senescent cells is their secretome called SASP (Coppé et al. 2008). Therefore, cells were exposed to 10 or $40 \mu \mathrm{M} \mathrm{SM}, 200 \mu \mathrm{M} \mathrm{H}_{2} \mathrm{O}_{2}$ or solvent control and at different time points thereafter the levels of secreted chemokines, cytokines and growth factors were determined by Bio-Plex assays. At each time point, 72 different factors were tested (Supplementary Fig. 5), and the fold regulation was calculated relative to the corresponding solvent controls. Since many factors were either secreted at low levels in general or the fold regulation was small, specific criteria were applied $(>20 \mathrm{pg} / \mathrm{mL}$ in combination with fold regulation $>2$ or $<-2$ ).

Figure 3 a shows that many factors were upregulated starting from day 14 after exposure. Therefore, the factors TECK, sTNF-R1, osteopontin (OPN), gp130, eotaxin-3 and 6Ckine were considered as late factors and some showed an upregulation over various timepoints and could, therefore, be considered as constant factors, e.g., MIF, MCP-1, IL-8, IL-6, Gro- $\alpha$, ENA-78 and BAFF. From the above-mentioned factors, some were upregulated already within $24 \mathrm{~h}$ or the first week after exposure, such as IL-8. Those could also additionally be considered as early factors. Moreover, the factors sTNF-R1, CXCL12 and eotaxin-3 seem to be upregulated in SM- but not in $\mathrm{H}_{2} \mathrm{O}_{2}$-induced senescent cells (Fig. 3b).

Some of the results were also confirmed on the mRNA level determined by qPCR (Fig. 3c, Supplementary Table 1). Therefore, mRNA was isolated from MSCs 21 days after exposure to 10 and $40 \mu \mathrm{M} \mathrm{SM}, 200 \mu \mathrm{M} \mathrm{H} \mathrm{H}_{2} \mathrm{O}_{2}$ or solvent. OPN (SPP1) was significantly upregulated more than threefold in $\mathrm{SM}$ and sixfold in $\mathrm{H}_{2} \mathrm{O}_{2}$, which is completely in line with the results described above. Similarly, also a twofold upregulation was observed for osteocalcin (BGLAP) in $40 \mu \mathrm{M} \mathrm{SM}$ and $\mathrm{H}_{2} \mathrm{O}_{2}$ exposed cells, gp130 (IL6ST) only in $\mathrm{H}_{2} \mathrm{O}_{2}$ and IL26, CCL1 and APRIL (TNFSF13) only in $40 \mu \mathrm{M}$ SM exposed cells. In addition to the secretome data, caspase 1 (CASP1) was determined to be twofold upregulated in $40 \mu \mathrm{M}$ SM.

Using another approach, the levels of over 270 cytokines, chemokines and growth factors were determined by a glassslide ELISA based array. Figure $3 \mathrm{~d}$ shows the more than twofold upregulated factors of senescent cells compared to control levels at 21 day post-exposure. The levels of osteoprotegerin (OPG) were found to be upregulated in all 
a
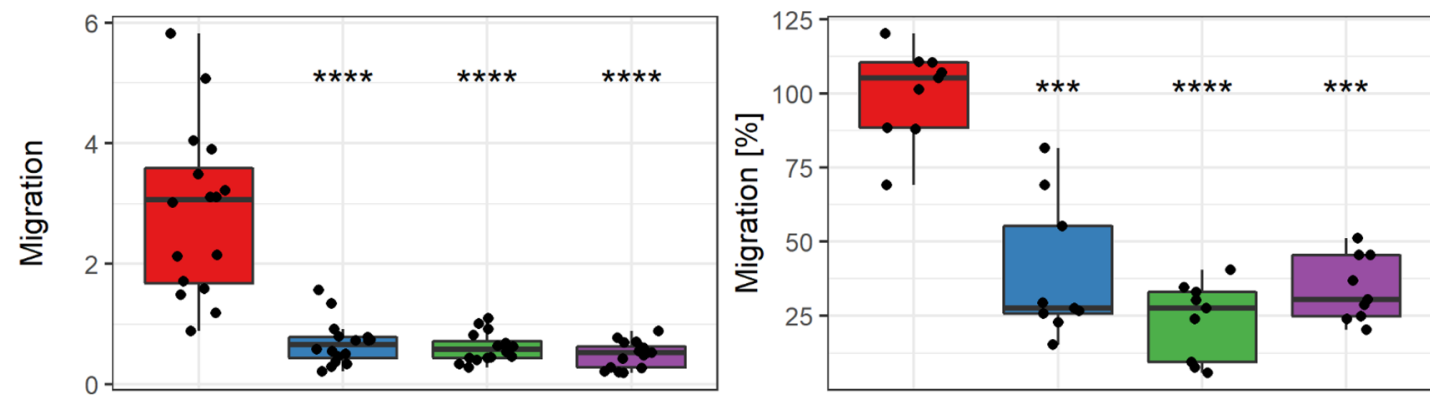

Cell type

b
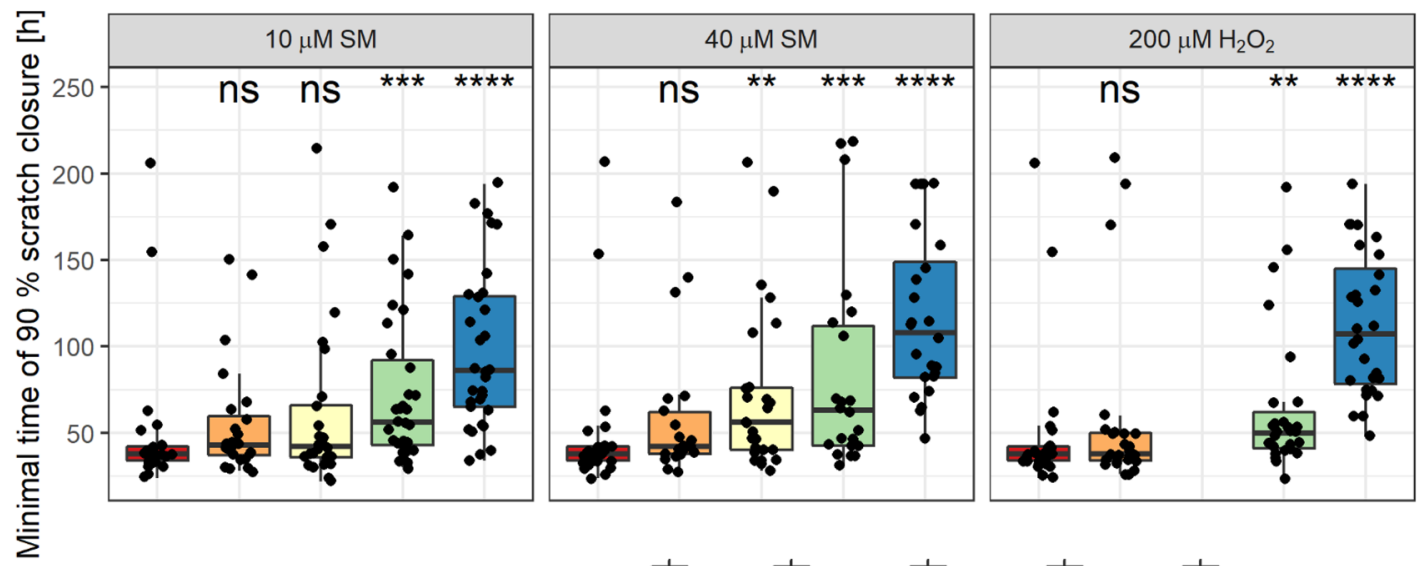

Percentage of senescent cells 0 \% 审 $10 \%$ 审 25\% 审 $50 \%$ 安 $100 \%$

C
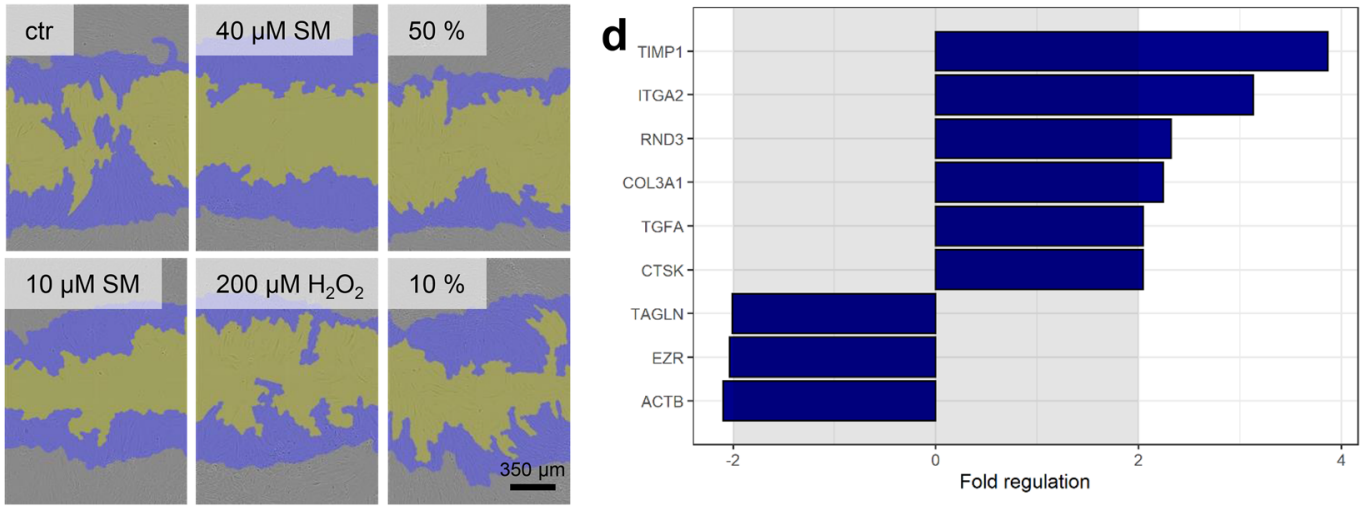

e
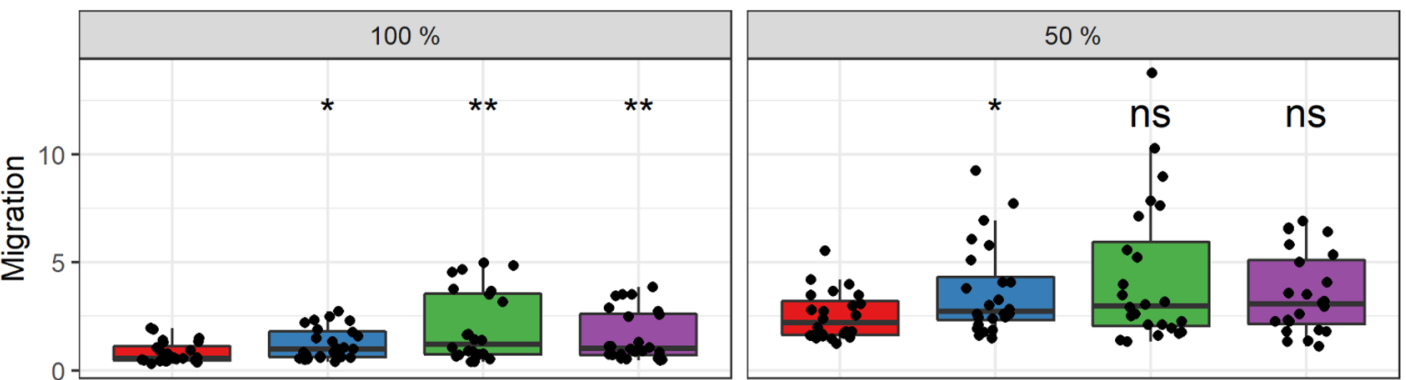

Conditioned medium

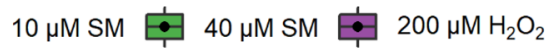

senescent cells. In both SM-induced senescent cell types IL-3 and adipsin were upregulated, while LH- $\beta$ and Dkk-4 were only upregulated in $\mathrm{H}_{2} \mathrm{O}_{2}$-induced senescence. In comparison, NCAM1, MMP2, M-CSF, IP-10, IL-7, IL-15, ferritin, 'Carcinoembryonic Antigen-Related Cell Adhesion Molecule 1 (CEACAM1)' and CEA are only upregulated 
४Fig. 4 Senescence-associated decreased migration and scratch closure. a Senescent cells showed a significant reduction in migration using IncuCyte migration (left) as well as modified Boyden chamber assay (right). Left: Different cells in FBS-reduced medium in inserts migrated towards the reservoir plate filled with standard medium. For normalization, the confluence on the bottom was divided by the confluence on the top for each time point and results are shown after $160 \mathrm{~h}$ ( $n=$ up to 8 biological replicates per group from three independent experiments). Right: Cells were added to the Boyden chamber and incubated for $8 \mathrm{~h}$, fixed, stained with DAPI, and migrated cells were counted. Migration was normalized to the mean of controls $(n=3$ biological replicates per group from three independent experiments). b, c Increasing amounts of senescent cells were added to non-senescent controls to observe the influence on scratch closure $(0 \%=$ only non-senescent solvent controls, $10 \%=10 \%$ senescent cells $+90 \%$ controls, and so on). b Scratch assay was performed using the wound maker and IncuCyte microscope, and the minimal time of $90 \%$ scratch closure was determined ( $n=$ up to 8 biological replicates per group from four independent experiments). c Representative images at $t=24 \mathrm{~h}$ (top right $50 \%$ and top bottom $10 \% 40 \mu \mathrm{M}$ $\mathrm{SM}$, blue initial scratch and yellow cell-free area). d Genes associated with wound healing and cell motility were tested in $40 \mu \mathrm{M} \mathrm{SM}$ exposed senescent and non-senescent cells. After RNA extraction from MSCs, a RT-qPCR assay was performed and fold regulation of up- or downregulated genes $(\geq 2.0$ or $\leq-2.0$ in combination with $p<0.05$, outside grey box) are shown as means ( $n=$ three independent experiments). e The migration of healthy MSCs was increased towards conditioned medium from senescent in comparison to that from non-senescent (control) cells. Conditioned medium was added into the reservoir plate (left) or diluted half and half with culture medium (right). For normalization, the confluence on the bottom was divided by the confluence on the top for each time point and results are shown after $188 \mathrm{~h}(\mathrm{n}=$ up to 8 biological replicates per group from three independent experiments). Data are represented as Tukey boxplots; $* p<0.05, * * p<0.01, * * * p<0.001, * * * * p<0.0001$. See also Supplementary Fig. 6

in $40 \mu \mathrm{M}$ SM senescent cells, but MMP10, MCP3, MCP2, IL-10 R $\alpha$ and activin A only in $10 \mu \mathrm{M}$ SM cells. Out of these, the MMP2- and IP-10 upregulation was similar to the results described above.

\section{Reduced migration and scratch closure ability of senescent cells and influence on healthy MSCs}

Important properties of MSCs in wound healing are their migration capability. To assess the migration potential, senescent and non-senescent MSCs were allowed to migrate using the chemotaxis assay by IncuCyte. Images were recorded every $2 \mathrm{~h}$ (Supplementary Fig. 6a) and for statistical analysis, the migration at the endpoint of the experiment was chosen and shown in Fig. 4a (left). Non-senescent controls showed a high migration potential of about 3 , which means three-fold more migrated cells than still present in the upper compartment. All senescent cells showed almost no migration at all, with fewer cells in the lower than the upper compartment $(<1)$, and was significant, when compared to controls. Using a slightly different technique, i.e., a modified Boyden chamber, migrated cells were counted after
8 h. Hereby also a significantly decreased migration was observed for the senescent cells in comparison to controls (Fig. 4a, right).

The scratch assay was used to study if senescent MSCs are still able to close a scratched area or "wound". Moreover, different percentages of senescent cells were added to non-senescent controls to see if they would change the time needed to close the scratch. For this assay, the wound maker tool from IncuCyte was used, images were recorded every $2 \mathrm{~h}$ and scratch closure was analyzed as the percentage of repopulated scratched area by cells (Supplementary Fig. 6b). For comparison, the minimal time to reach a $90 \%$ scratch closure was calculated and results are shown in Fig. 4b, c. Between the non-senescent control ( $0 \%$ senescent cells) with a minimal time of $49.4 \pm 40.9 \mathrm{~h}$ and the senescent cells ( $100 \%$ senescent cells) with $97.5 \pm 46.4 \mathrm{~h}$ for $10 \mu \mathrm{M} \mathrm{SM}$, $116 \pm 47 \mathrm{~h}$ for $40 \mu \mathrm{M} \mathrm{SM}$ and $112 \pm 41 \mathrm{~h}$ for $200 \mu \mathrm{M} \mathrm{H}_{2} \mathrm{O}_{2}$ the scratch closure times were significantly elongated by about twofold. The mixture of $90 \%$ controls with $10 \%$ senescent cells did not result in a significant increase in the minimal time, but with increasing percentages of senescent cells, the minimal time increased. This increase was significant for the 50:50 mixture of all senescent cells with controls and for $25 \% 40 \mu \mathrm{M}$ SM with $75 \%$ control.

To understand the underlying mechanisms of this migration and scratch closure reduction, gene expression related to cell motility and wound healing was evaluated by RT-qPCR (Fig. 4d, Supplementary Table 1). Wound healing related genes besides COL3A1 (see 2.2) such as 'TIMP metallopeptidase inhibitor 1 (TIMP1)'; 'integrin, $\alpha 2$ (ITGA2)'; 'transforming growth factor, $\alpha$ (TGFA)' and 'cathepsin K (CTSK)' were upregulated between 2 - and almost fourfold in $40 \mu \mathrm{M}$ SM senescent cells compared to controls. The motility related 'Rho family GTPase 3 (RND3)' was upregulated twofold. Downregulated by more than twofold were 'transgelin (TAGLN)'; 'ezrin (EZR)' and 'actin, beta (ACTB)'.

Senescent cells secrete a variety of proinflammatory factors and their conditioned medium increased the migration of tumor cell lines (Minieri et al. 2015). Thus, the influence of conditioned medium onto the migration of healthy lowpassage MSCs was studied using the IncuCyte microscope (Supplementary Fig. 6c). For statistical analysis, migration at the endpoint was chosen and shown in Fig. 4e. The migration towards pure conditioned medium (100\%, left side) was significantly higher when derived from all senescent cells in comparison to non-senescent controls. To reduce a possible nutrient deficiency in the conditioned medium, it was mixed with the same volume of fresh medium $(50 \%$, right side). Using this set-up, only the conditioned medium from $10 \mu \mathrm{M}$ SM senescent cells resulted in a significant increase compared to medium from controls, but for all a tendency towards an increased migration could be shown. 


\section{Elimination with senolytic drugs}

Since senescent cells display various detrimental properties, their targeted and specific elimination might result in improved wound healing, as shown before (Demaria et al. 2014). A total of eight different drugs described as senolytics in the literature were used in increasing concentrations to determine the half-maximal lethal concentration $\left(\mathrm{LC}_{50}\right)$ in senescent (10 and $40 \mu \mathrm{M} \mathrm{SM}$ or $200 \mu \mathrm{M} \mathrm{H}_{2} \mathrm{O}_{2}$ ) and nonsenescent control MSCs. The senolytics applied were from different groups. Etomoxir and antimycin A target differences between senescent and non-senescent cells in cell metabolism (Dörr et al. 2013); dasatinib and quercetin are kinase inhibitors (Zhu et al. 2015); ABT-737 and ABT263 are inhibitors of anti-apoptotic BCL proteins (Chang et al. 2016; Yosef et al. 2016); FOXO4-DRI is a synthetic peptide which inhibits the interaction between FOXO4 and p53 (Baar et al. 2017); and 17-DMAG is a HSP90 inhibitor (Fuhrmann-Stroissnigg et al. 2017). First, viability was tested 5 day after the exposure to senolytics and the $\mathrm{LC}_{50}$ values including $99 \%$ confidence intervals are shown in Table 1. None of the tested drugs showed the desired specificity, while ABT-263, antimycin A, etomoxir and quercetin seemed to affect all cells almost equally with mostly overlapping confidence intervals around the $\mathrm{LC}_{50}$. By contrast, the other tested substances, i.e., 17-DMAG, dasatinib, ABT-737 and FOXO4-DRI, displayed a higher specificity for non-senescent cells, based on the lower $\mathrm{LC}_{50}$ value than senescent cells. In our hands, ABT-737 was not even able to reduce the viability of senescent cells until the limit of solubility was reached. Since senolytics might not be able to persist and act for as long as 5 days, a shorter time period of $24 \mathrm{~h}$ was additionally used for three selected substances and the reduced time span resulted in a tolerance to overall higher concentrations. The substances 17-DMAG and dasatinib showed a comparable result as the non-senescent controls were still more affected. Surprisingly, the reduced time span resulted in the desired effect for ABT-263 with a significantly reduced $\mathrm{LC}_{50}$ value for all three senescent cell types compared to non-senescent controls (Table 1). The comparison between the time points is shown in Fig. 5 and other senolytics in Supplmentary Fig. 7.

\section{Discussion}

Here we provide insight into a novel pathomechanism after SM exposure, the chronic senescence in human MSCs as possible contribution to the chronic wound healing disorder. Our results show a concentration- and time-dependent senescence induction after single dose SM exposure with deep senescence from 10 to $40 \mu \mathrm{M}$ SM 21 days later, recorded as intense SA- $\beta$-gal staining of all cells. This senescence was chronic for $40 \mu \mathrm{M} \mathrm{SM}$ induced MSCs, since they were still fully senescent even 24 weeks after exposure. As a proof of principle, senescence could also be induced in commercially available adipose tissue derived MSCs. Moreover, senescence could be induced by continuous treatment with lower SM concentrations, and the senescence phenotype by SA- $\beta$-gal staining was comparable to MSCs exposed to alkylating cytostatic drugs (Collado et al. 2007) as well as to ionizing radiation (Alessio et al. 2015) in accordance with published studies. For comparison, solvent controls were used as non-senescent MSCs for further studies, since they kept their replicative potential and showed replicative senescence only at passages way higher than those used in experiments, and exposure to $200 \mu \mathrm{M} \mathrm{H}_{2} \mathrm{O}_{2}$ was used as a positive senescence induction control (Brandl et al. 2011). Our results also suggest that SM exposure of MSCs results primarily in senescence for lower concentrations (up to $40 \mu \mathrm{M}$ ), while higher concentrations mainly lead to apoptosis, as determined by annexin $\mathrm{V}$. This is in line with previous findings of our group revealing that likewise, up to $40 \mu \mathrm{M}$
Table $1 \mathrm{LC}_{50}$ values for senolytic drugs. $\mathrm{LC}_{50}$ values including the $99 \%$ confidence intervals for senolytic drugs after treatment for 5 days and $24 \mathrm{~h}$

\begin{tabular}{llllll}
\hline Drug & Treatment & Control & $10 \mu \mathrm{M} \mathrm{SM}$ & $40 \mu \mathrm{M} \mathrm{SM}$ & $200 \mu \mathrm{M} \mathrm{H}_{2} \mathrm{O}_{2}$ \\
\hline 17-DMAG $[\mathrm{nM}]$ & 5 days & $118 \pm 13$ & $460 \pm 66$ & $510 \pm 68$ & $1911 \pm 148$ \\
17-DMAG $[\mathrm{nM}]$ & $24 \mathrm{~h}$ & $31,041 \pm 2573$ & $59,457 \pm 6661$ & $44,276 \pm 5,408$ & $42,871 \pm 3036$ \\
ABT-263 $[\mu \mathrm{M}]$ & 5 days & $31.6 \pm 0.5$ & $36.1 \pm 2.2$ & $33.8 \pm 1.5$ & $33.1 \pm 0.3$ \\
ABT-263 $[\mu \mathrm{M}]$ & $24 \mathrm{~h}$ & $58.1 \pm 1.6$ & $48.9 \pm 0.9$ & $43.4 \pm 1.2$ & $41.7 \pm 0.9$ \\
ABT-737 $[\mu \mathrm{M}]$ & 5 days & $115 \pm 11$ & $(1054 \pm 315)$ & $(2,364 \pm 1,403)$ & $(8536 \pm 12,628)$ \\
Antimycin A $[\mu \mathrm{M}]$ & 5 days & $560.9 \pm 92.3$ & $513.3 \pm 39.8$ & $613.3 \pm 61.5$ & $586.4 \pm 99.6$ \\
Dasatinib $[\mathrm{nM}]$ & 5 days & $152 \pm 25$ & $2259 \pm 362$ & $3692 \pm 742$ & $1543 \pm 604$ \\
Dasatinib $[\mathrm{nM}]$ & $24 \mathrm{~h}$ & $1690 \pm 813$ & $20,773 \pm 7103$ & $11,633 \pm 4131$ & $24,457 \pm 8867$ \\
Etomoxir $[\mu \mathrm{M}]$ & 5 days & $544 \pm 21$ & $568 \pm 17$ & $584 \pm 17$ & $555 \pm 23$ \\
FOXO4-DRI $[\mu \mathrm{M}]$ & 5 days & $44.4 \pm 1.8$ & $53.1 \pm 2.3$ & $57.7 \pm 2.1$ & $56.4 \pm 2.3$ \\
Quercetin $[\mu \mathrm{M}]$ & 5 days & $116.0 \pm 8.7$ & $104.1 \pm 5.4$ & $121.1 \pm 7.6$ & $177.2 \pm 10.6$ \\
\hline
\end{tabular}

Italic values in brakes reflect calculations but are above solubility limit 


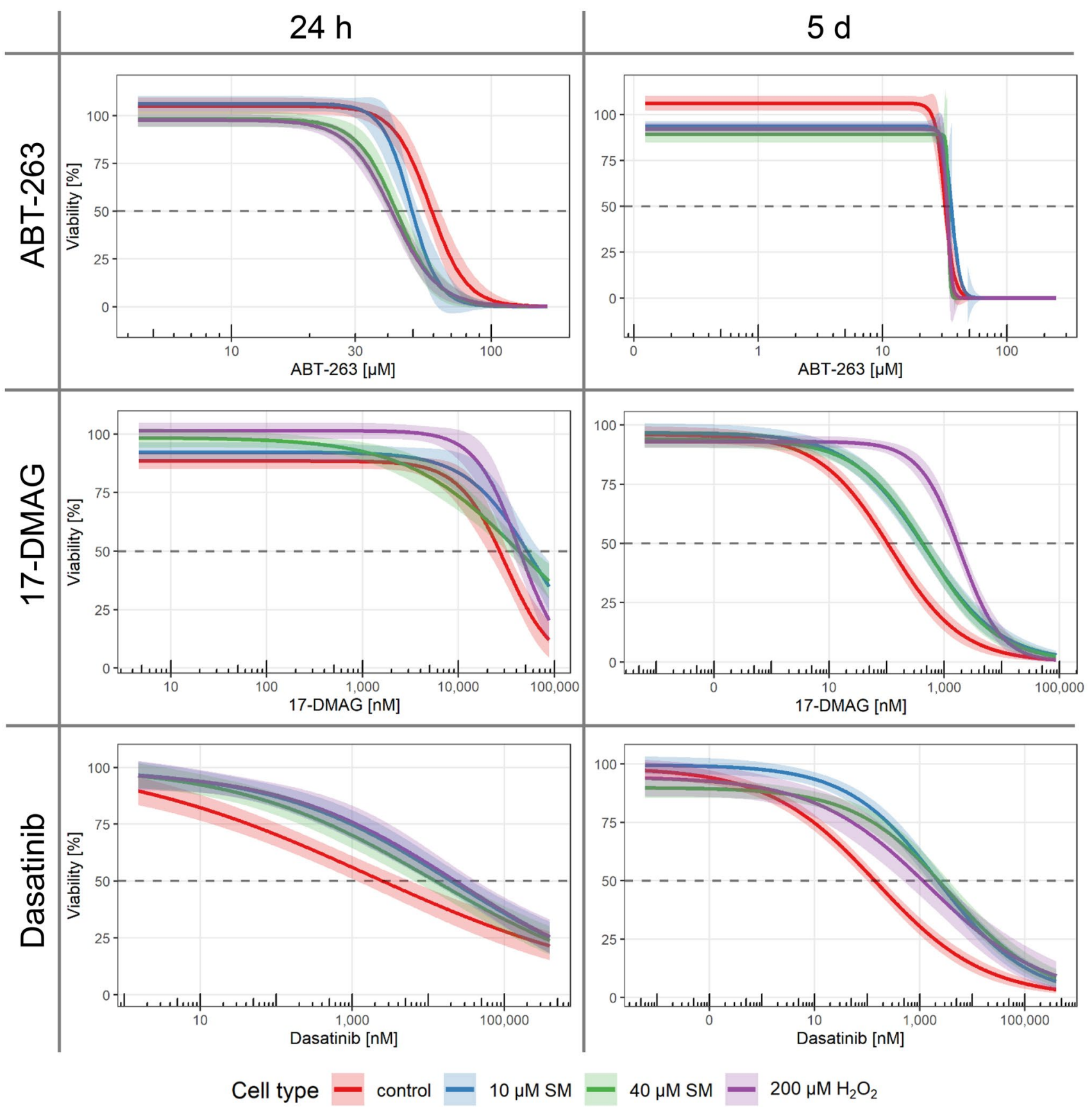

Fig. 5 Elimination with senolytics. The selectivity towards senescent cells of literature reported senolytic drugs was tested. Senescent and non-senescent (control) MSC were treated with increasing concentrations of ABT-263, 17-DMAG or dasatinib for 5 days or $24 \mathrm{~h}$. Viability was assessed by XTT assay and normalized for each cell type

SM exposure did not elevate levels of cleaved caspase-3, caspase-8, PARP p85 and apoptosis-inducing factor within $48 \mathrm{~h}$ (Schmidt et al. 2018a). In contrast, $\mathrm{H}_{2} \mathrm{O}_{2}$ exposure resulted in both senescence and apoptosis for the applied concentration, already suggesting differences between SMand $\mathrm{H}_{2} \mathrm{O}_{2}$-induced senescence. to the corresponding solvent controls ( $n=4$ biological replicates per group from two or three independent experiments). Data are represented as linear regression including $99 \%$ confidence intervals. See also Supplementary Fig. 7

Since senescence was already shown to be induced by unresolved DNA damage (Sedelnikova et al. 2004) or RO S (von Zglinicki 2002), it is not surprising that SM also leads to senescence, as it results in various DNA and RNA monoalkylation adducts and crosslinks (Zubel et al. 2019) as well as the formation of oxygen free radicals (Brimfield et al. 
2012). Moreover, first hints of senescence are described as reduced telomere length in SM exposed Iranian veterans (Behboudi et al. 2018; Behravan et al. 2018) and our group has already discovered acute senescence after SM treatment (Schmidt et al. 2018a). Concluding that the blisterinducing concentration of SM is about $150 \mu \mathrm{M}$ (Smith et al. 1993 ) and only $16 \%$ reaches the circulation (Cullumbine 1947), this would result in low plasma levels. Even $10 \mu \mathrm{M}$ SM induced a chronic senescence in MSCs, which highlights a possible transferability of our results to in vivo scenarios.

The chronic senescence induced by SM was substantiated by various biomarkers. SA- $\beta$-gal positive cells could be easily determined by flow cytometry using a fluorogenic substrate, a convenient and unbiased technique. Higher area and side scatter values are in line with the observed increase in cell size and granularity already during culture and $\mathrm{H} / \mathrm{E}$ staining. Our results also show loss of proliferation in senescent cells determined by reduced colony forming potential. Significantly elevated levels of $\mathrm{p} 16^{\mathrm{INK} 4 \mathrm{~A}}$ were detected both on the protein and mRNA level, a key regulator of in vitro senescence in human cells. It is absent in unstressed tissues of young animals but highly expressed after certain stresses occurring with tumorigenesis, wounding or aging (Sharpless and Sherr 2015). p21 also showed an increase, which was, however, only significant on mRNA level. The chronic senescence after SM exposure seems to be maintained by a specific upregulation, including also CDKN2B, MDM2, CREG1, COL3A1, SPARC and GADD45A, or downregulation, including CHEK1, CDK2, CDKN2C, CCNA2, CCNB1 and RBL1, of cell cycle regulators. Additionally, the persistently upregulated DDR was found in senescent MSCs before (Minieri et al. 2015), which is in line with our results. Almost all DNA repair mechanisms showed differences, but interestingly, most affected genes seem to be involved in the homologous recombination repair of DNA double strand breaks including XRCC2 and EXO1 upregulation and H2AFX, RAD51, BRCA1, BARD1 and BRIP1 downregulation. This gene expression pattern may include some differences to other senescent cells, which would provide interesting new insight into SM-induced senescence in MSCs.

Another essential biomarker is SASP, a term for the senescence-specific secretome (Coppé et al. 2008). The quality and quantity of SASP factors show differences between cell lines, tissues, species, initial senescence inducing strategies and time after senescence induction (Borodkina et al. 2018). Not only cellular senescence itself but also the SASP is hypothesized to be a dynamic process, divided into (1) acute factors (within $36 \mathrm{~h}$ ), (2) early phase (initiation of senescence up to 10 days later) and (3) the chronic phase ("mature SASP", after 2-3 weeks). The secreted factors are thought to create a proinflammatory tissue environment as a paracrine effect and reinforce senescence by autocrine effects, which is influenced by positive feedback loops and various complex regulatory mechanisms (Malaquin et al. 2016; Borodkina et al. 2018). Our results clearly show the upregulation of various proinflammatory factors following senescence development. TECK, sTNF-R1, OPN, gp130, eotaxin-3 and 6Ckine could be considered as late factors. Moreover, MIF, MCP-1, IL-8, IL-6, Gro- $\alpha$, ENA-78 and BAFF could be considered as constant factors, since they were upregulated during various time points. Out of the factors considered as SASP core for any cell undergoing senescence (Coppé et al. 2008), IL-6, IL-8, Gro- $\alpha$ and MCP-1 but not GM-CSF were also found upregulated in our study. Our results do not confirm GM-CSF as a SASP core factor, which may result from SM as a novel senescence inducer or by human MSCs used as cell type. The absent upregulation of anti-inflammatory cytokines like IL-4, IL-10, IL-13 or IL-35, as typically seen in SASP, is also in line with literature data (Byun et al. 2015). The late factors are of special interest, since they correlate with deep or chronic senescence. For example, OPN is important in immune functions acting as an immune modulator to promote cell recruitment to inflammatory sites, as an adhesion protein involved in cell attachment and wound healing, mediating cell activation and cytokine production, and promoting cell survival as an antiapoptotic factor (Wang and Denhardt 2008). Moreover, the three factors sTNF-R1, CXCL12 and eotaxin-3 seem to be upregulated in SM-induced, but not in $\mathrm{H}_{2} \mathrm{O}_{2}$-induced senescent cells, which makes them the most interesting factors determined in our study. While sTNF-R1 and eotaxin-3 are not yet described as SASP factors, the literature already displays an importance of CXCL12 in MSCs, senescence and wound healing. CXCL12 is important in angiogenesis and inflammation, and suppression of its activity was suggested to improve scar-free wound healing (Willyard 2018). It is constitutively expressed by MSCs, where it is responsible for the retention of hematopoietic progenitor and stem cells (Janssens et al. 2018) and CXCL12 was largely expressed in senescent tumor cells, where it was related to cancer cell migration and metastasis (Kim et al. 2017). Further research is needed to prove our findings and unravel the underlying mechanisms, eventually resulting in new targets for SM therapy.

Our results show that senescent MSCs have a significantly reduced migratory ability, which was also seen in irradiation induced senescent MSCs (Carlos Sepúlveda et al. 2014). Migration of MSCs is a very important characteristic, since it is essential for homing to damaged tissue to trigger wound healing in vivo (Wang et al. 2014) and this reduction may explain part of its disturbance after SM exposure. Supporting this, the reduced migration resulted in decreased scratch closure ability modeling the wound healing in vitro. Our results showed not only that $\mathrm{SM}$ or $\mathrm{H}_{2} \mathrm{O}_{2}$ induced senescent MSCs needed significantly more time to close the scratch, 
but also the mixture of 50\% senescent with 50\% non-senescent cells resulted in a significant increase in scratch closure time. This might imply that even a fraction of senescent MSCs might be sufficient to result in the wound healing disorder, since it is unrealistic that all MSCs of an SM exposed victim would become senescent. A panel of cell motility and wound healing genes was analyzed to gain more insight into the underlying mechanisms. In extracellular matrix participating mRNAs of TIMP1, integrin $\alpha-2$, TGF- $\alpha$, COL3A1 and cathepsin $\mathrm{K}$ were upregulated. The reformation and reorganization of extracellular matrix during wound healing is essential, for example by providing a provisional matrix for the migration of keratinocytes (Thiruvoth et al. 2015). Genes involved in the actin cytoskeleton also seem to play a role in SM induced senescence, since ezrin, its linker to the plasma membrane, the cross-linking transgelin as well as $\beta$-actin itself were found to be downregulated, while Rnd3, the regulator in response to extracellular growth factors, was upregulated. Actin filaments as major cell cytoskeleton play important roles in wound healing in vivo, by establishing the initial plug of the wound, where it accumulates at the wound edge, and during remodeling (Abreu-Blanco et al. 2012).

The conditioned medium of senescent cells containing the SASP factors in general and of MSCs in particular was shown to significantly enhance the migration of solid tumor-derived cell lines (Minieri et al. 2015). In our study, we found that the conditioned medium of senescent cells, in contrast to conditioned medium of control cells, enhanced the migration of 'healthy' early-passage MSCs. First, this may seem beneficial, since migration is a key property of MSCs (Wang et al. 2014), but on a closer look the healthy MSCs may be more attracted to home to senescent cells than to the site of tissue injury, therefore, resulting in prolonged or disabled wound healing. Moreover, a paracrine transmission of senescence is proposed (van Deursen 2014), already shown for replicative senescent MSCs (Severino et al. 2013), and thus healthy MSCs might also turn senescent by the 'bystander effect' (Nelson et al. 2012) resulting in a negative feedback loop.

Taken together, all these detrimental effects of SMinduced MSCs might contribute to the wound healing disorder. Therefore, a treatment using senolytic drugs to selectively eliminate such senescent MSCs would be a novel treatment option. Recent studies raised hopes for regenerative medicine or aging, since the application of senolytic agents was successfully performed in animal studies and intermittent short treatments will have low to no risk of side effects, because senescent cells are unlikely to develop drug resistance as they do not divide (Tchkonia et al. 2013; Zhu et al. 2015). Moreover, senescent cells are increasingly linked to chronic wounds and application of senolytics is discussed as a possible treatment option (Wilkinson and Hardman 2020). In our study, none of the eight senolytic drugs antimycin A, etomoxir, dasatinib, quercetin, ABT-263, ABT-737, FOXO4-DRI and 17-DMAG showed the expected senescence specific effect on cell viability when applied over 5 days. Since ABT-263 exposure of 1 day revealed a senolytic effect in MSCs (Grezella et al. 2018), we additionally tested ABT-263 as well as 17-DMAG and dasatinib with a $24 \mathrm{~h}$ exposure. While 17-DMAG and dasatinib were comparable to the longer exposure, ABT-263 exposure of $24 \mathrm{~h}$ resulted in the expected lower $\mathrm{LC}_{50}$ values of senescent in contrast to non-senescent MSCs. We, therefore, propose ABT-263 as a possible drug candidate.

In conclusion, we demonstrated chronic senescence in human MSCs already after SM single dose exposure. These are impaired regarding proliferation and migration, secrete a variety of proinflammatory cytokines, possibly attract healthy MSCs and may render them senescent. The SM induced senescent MSCs, unable to fulfil their regenerative role, may contribute to the wound healing disorder. An innovative treatment strategy for SM might be the selective clearance of senescent cells by senolytic drugs, an approach in which ABT-263 showed initial potential. Moreover, due to the mechanistic similarity of SM with alkylating cytostatic drugs, our results may also be of interest for therapy-induced senescence (TIS) in cancer patients as well as for the treatment of age-related diseases.

\section{Materials and methods}

\section{Isolation and subcultivation of MSCs}

Human mesenchymal stem cells (hMSCs, henceforth termed MSCs) were isolated from bone marrow of femoral heads, which were donated in the context of total endoprosthesis of the hip joint. They were obtained after obtaining informed consent from patients at the HELIOS Amper Hospital in Dachau, Germany, or Wolfart Hospital in Gräfelfing, Germany. All experiments with human cell material was performed according to authorization of the ethics committee of Ludwig Maximilian University Munich, Germany, and treated as well as disposed according to the requirements of a BioSafety II laboratory. For the experiments, 99 femoral heads were used from donors between 50 and 89 years (mean age 71 years; $46 \%$ male and $54 \%$ female).

Bone marrow was scraped from the femoral heads using a sharp curette (Allgaier Instrumente $\mathrm{GmbH}$, Frittlingen, Germany), and MSCs were released from trabecular bones by agitation into stem cell medium (HyClone ${ }^{\mathrm{TM}}$ minimum essential medium [MEM] alpha modification [GE Healthcare Bio-Sciences Austria GmbH, Pasching, Austria], 20\% $[\mathrm{v} / \mathrm{v}$ ] fetal bovine serum [FBS], $400 \mu \mathrm{M}$ L-glutamine, 100 $\mathrm{U} / \mathrm{mL}$ penicillin, $100 \mathrm{U} / \mathrm{mL}$ streptomycin [all Gibco ${ }^{\circledR}$ by Thermo Fisher Scientific, Waltham, USA]). For separation, 
the suspension was filtered through a $70 \mu \mathrm{m}$ cell strainer (BD Falcon ${ }^{\mathrm{TM}}$ by Corning, Inc., Corning, USA), and a density gradient centrifugation was performed using Ficoll-Paque ${ }^{\mathrm{TM}}$ Plus (GE Healthcare, Chicago, USA) overlaid with the cell suspension for $30 \mathrm{~min}$ at $660 \times \mathrm{g}$, with the brake switched off. MSCs were enriched in the interphase, which was washed once with stem cell medium and centrifuged at $522 \times g$ for $5 \mathrm{~min}$ and plated onto $90 \mathrm{~mm}$ cell culture dishes (VWR International, Radnor, USA). Cells were incubated at $37^{\circ} \mathrm{C}$ in a humidified atmosphere containing $5 \% \mathrm{CO}_{2}$ (Thermo Fisher Scientific, Waltham, USA). After 2 days, a medium exchange was performed to eliminate hematopoietic cells. MSCs were further cultured and passaged at about $60 \%$ confluence.

Passaging was performed with StemPro ${ }^{\circledR}$ Accutase ${ }^{\circledR}$ Cell Dissociation Reagent (Life Technologies, Carlsbad, USA) for $5 \mathrm{~min}$ in the incubator after washing once with PBS. The cell suspension was diluted in medium, pelleted at $522 \times \mathrm{g}$ for $5 \mathrm{~min}$, resuspended in fresh medium and counted with Neubauer improved counting chamber (NanoEnTek Inc, Seoul, Korea). Cells were replated at 2000 cells per $\mathrm{cm}^{2}$ in fresh medium. Start of experiments was performed up to passage \#3. To obtain the best possible comparability, cells were never frozen but instead always used fresh, and plastic labware as well as medium components were kept constant.

\section{Identification of isolated MSCs}

Cells were stained with five different cell surface markers and analyzed via flow cytometry. Briefly, a suspension of 50,000 to 500,000 cells of a low passage number in $1 \mathrm{~mL}$ culture medium was stained with the following labeled antibodies for $15 \mathrm{~min}$ at room temperature: CD14-FITC (5 $\mu \mathrm{L})$, CD34-PE-Cy7 (1 $\mu \mathrm{L})$, CD45-APC-Cy7 (1 $\mu \mathrm{L})$, CD105PerCP-Cy5.5 $(1 \mu \mathrm{L})$ and CD106-APC $(5 \mu \mathrm{L}$; all Becton Dickinson, Franklin Lakes, USA). An unstained as well as an isotype control using the following labeled antibodies was included: IgG2 $\kappa$-FITC $(5 \mu \mathrm{L}), \operatorname{IgG} 1 \kappa-P E-C y 7(5 \mu \mathrm{L})$,

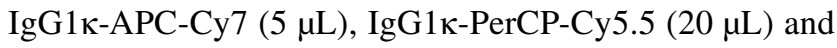

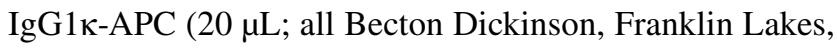
USA). After staining, cell suspension was washed once, resuspended in annexin binding buffer (Becton Dickinson, Franklin Lakes, USA) and analyzed with BD FACSCANTO Flow Cytometer (Becton Dickinson, Franklin Lakes, USA). MSCs are defined as $\mathrm{CD} 14^{-} / \mathrm{CD} 34^{-} / \mathrm{CD} 45^{-} / \mathrm{CD} 105^{+} /$ CD106 ${ }^{+}$.

Moreover, MSCs were also characterized by their potential to differentiate into osteocytes and adipocytes. Therefore, $3.15 \times 10^{4}$ cells per $\mathrm{cm}^{2}$ were seeded onto coverslips in 4-well plates. Differentiation medium (PromoCell, Heidelberg, Germany) was changed every $2-3$ days for 21 days for osteogenic and 14 days for adipogenic differentiation, respectively. Calcium-rich areas were stained with alizarin red S (Sigma-Aldrich, St. Louis, USA) and lipid drops with Sudan-III (Bio-Optica, Milano, Italy), both with hematoxylin nuclear staining (Bio-Optica, Milano, Italy).

\section{Viability assessment after hydrogen peroxide exposure}

MSCs were plated at 40,000 cells per well in two 24-well plates (Greiner AG, Kremsmünster, Austria) including medium control and grown overnight. The next day, cells were exposed to increasing concentrations of $\mathrm{H}_{2} \mathrm{O}_{2}$ $(0.2-80,000 \mu \mathrm{M})$ as well as solvent control for 5 days. The $30 \%$ (w/w) $\mathrm{H}_{2} \mathrm{O}_{2}$ solution in $\mathrm{H}_{2} \mathrm{O}$ (Sigma-Aldrich, St. Louis, USA) was pre-diluted in ultra-pure water and finally diluted in culture medium. Afterwards cells were washed once with PBS and XTT staining solution (Sigma-Aldrich, St. Louis, USA) was prepared by mixing $5 \mathrm{~mL}$ XTT labeling reagent with $100 \mu \mathrm{L}$ electron-coupling reagent per plate. Cells were incubated with $400 \mu \mathrm{L}$ medium and $200 \mu \mathrm{L}$ XTT staining solution, and absorbance was determined at $450 \mathrm{~nm}$ with a reference set at $630 \mathrm{~nm}$. Background absorbance was removed using wells only containing medium and viability was normalized to solvent controls. This experiment was performed six times independently (i.e., with cells obtained from six individual donor materials).

\section{Induction of senescence by sulfur mustard and hydrogen peroxide exposure}

Cells were used up to passage three for senescence induction and up to 7 days after last plating (about $70 \%$ confluence). Therefore, cells were grown in T175 flasks (Greiner AG, Kremsmünster, Austria).

SM (bis-[2-chloroethyl]sulfide; purity $>99 \%$, confirmed by NMR) was made available by the German Ministry of Defense. For the initial senescence induction study, SM concentrations of $1,10,20$ and $40 \mu \mathrm{M}$, while for later experiments only 10 and $40 \mu \mathrm{M}$ were used. SM was pre-diluted in pure EtOH and finally diluted in culture medium with a maximum of $0.5 \% \mathrm{EtOH}$. Solvent controls were treated with the same volume of pure EtOH. $\mathrm{H}_{2} \mathrm{O}_{2}$ was used as positive senescence control at $200 \mu \mathrm{M}$ final concentration. The $30 \%$ (w/w) $\mathrm{H}_{2} \mathrm{O}_{2}$ solution in $\mathrm{H}_{2} \mathrm{O}$ (Sigma-Aldrich, St. Louis, USA) was once pre-diluted and finally diluted both in culture medium.

For $\mathrm{SM}$ and $\mathrm{H}_{2} \mathrm{O}_{2}$ exposed cells, culture medium was changed once a week without passaging of the cells. Solvent controls were sub-cultured once a week by plating 2000 cells per $\mathrm{cm}^{2}$. Senescent (10 $\mu$ M SM, $40 \mu \mathrm{M}$ SM and $200 \mu \mathrm{M}$ $\mathrm{H}_{2} \mathrm{O}_{2}$ ) and non-senescent cells (solvent control) were used for further experiments 21 days after exposure unless otherwise stated. 


\section{Time- and concentration dependence of senescence induction by $S A-\beta-g a l$ staining}

After exposure to 1, 10, 20 and $40 \mu \mathrm{M} \mathrm{SM}$ or $200 \mu \mathrm{M} \mathrm{H}_{2} \mathrm{O}_{2}$, senescence development was checked every third to fourth day using the senescence detection kit I (PromoCell, Heidelberg, Germany) according to manufacturer's protocol. To exclude false positive staining, cells were plated at the same density 1 day before staining onto cover slips in 4-well plates. Briefly, 10,000 cells were grown overnight on coverslips in 4-well plates, washed once with PBS and fixed for 10-15 min at room temperature with the fixative solution. Meanwhile, the senescence staining solution was prepared by mixing $470 \mu \mathrm{L}$ of staining solution, $5 \mu \mathrm{L}$ of staining supplement and $25 \mu \mathrm{L}$ of $20 \mathrm{mg} / \mathrm{mL}$ X-gal in DMSO per well. Fixed cells were washed twice with PBS and senescence staining solution was added and developed in the incubator overnight. Additionally, cells were counterstained with nuclear fast red (Vector Laboratories, Inc., Burlingame, USA) for $10 \mathrm{~min}$ at room temperature and mounted with VectaMount ${ }^{\mathrm{TM}}$ AQ mounting medium (Vector Laboratories, Inc., Burlingame, USA) onto microscopy slides (Thermo Fisher Scientific, Waltham, USA). Senescence development was monitored over a time period of 4 weeks. The percentage of senescent versus total cells was counted in three randomly selected images (mean of 20 cells per image) and was performed in three independent experiments.

\section{Replicative senescence}

To assess replicative senescence, MSCs were re-plated at 2000 cells per $\mathrm{cm}^{2}$ once a week in flasks or culture plates and onto coverslips for SA- $\beta$-gal staining (see above). This was performed until the cells did not replicate anymore and no more sub-cultivation was possible, since all cells were used for staining. Experiment was performed three times independently.

\section{Stability of SM- and $\mathrm{H}_{2} \mathrm{O}_{2}$-induced senescence}

The stability of the induced senescence by 10 and $40 \mu \mathrm{M}$ SM as well as $200 \mu \mathrm{M} \mathrm{H}_{2} \mathrm{O}_{2}$ was determined by SA- $\beta$-gal staining each 5 weeks. Therefore, medium exchange was performed once a week, and every 5 week cells were passaged and 10,000 cells each were used for staining, while the remaining cells were further cultivated. The last staining was performed 24 weeks after exposure and experiment was performed three times independently.

\section{Time- and concentration dependence of senescence induction in commercially available MSCs}

The same experiment as described in 4.5 was performed with commercially available normal human adipose-derived
MSCs and the recommended MSC basal medium plus MSC growth kit-Low serum (all from American Type Culture Collection [ATCC], Manassas, USA). Briefly, one vial was thawed, and cells were plated at 2000 cells per $\mathrm{cm}^{2}$ and subcultured once a week using the same procedure as for bone marrow derived MSCs. After exposure to solvent control, 10 and $40 \mu \mathrm{M}$ SM or $200 \mu \mathrm{M} \mathrm{H}_{2} \mathrm{O}_{2}$, SA- $\beta$-gal staining was performed every 3-4 days up to day 31 . Experiment was performed two times independently.

\section{Senescence induction by continuous exposure and other senescence inducers}

It was also investigated whether continuous $\mathrm{SM}$ and $\mathrm{H}_{2} \mathrm{O}_{2}$ treatment induced senescence. Therefore, MSCs were exposed to $0.1 \mu \mathrm{M}, 0.5 \mu \mathrm{M}$ and $1 \mu \mathrm{M}$ SM as well as $50 \mu \mathrm{M}$ $\mathrm{H}_{2} \mathrm{O}_{2}$ two to three times a week and re-plated for sub-cultivated and SA- $\beta$-gal staining once a week up to day 42 . Experiment was performed two times independently.

MSCs were exposed to $20 \mu \mathrm{M}$ cisplatin, $50 \mu \mathrm{M}$ melphalan or $50 \mu \mathrm{M}$ bendamustine (all Sigma-Aldrich, St. Louis, USA). Stock solutions of $40 \mathrm{mM}$ cisplatin in DMSO, $33 \mathrm{mM}$ melphalan in acidic EtOH and $40 \mathrm{mM}$ bendamustine in ultrapure water were prepared and then diluted in culture medium. Cells were irradiated at RT with a dose rate of 1 Gy per min with $240 \mathrm{kV}$ x-rays at $13 \mathrm{~mA}$ (YXLON Maxishot, Hamburg, Germany) filtered with $3 \mathrm{~mm}$ beryllium for $10 \mathrm{~min}$ (10 Gy). SA- $\beta$-gal staining was performed twice a week up to day 23 and the experiment was performed two times independently.

\section{Confluence and apoptosis}

Cells were plated at 8000 cells per well in a 24 -well plate and incubated overnight. The next day, cells were exposed to solvent or SM concentrations of $10 \mu \mathrm{M}, 40 \mu \mathrm{M}, 63 \mu \mathrm{M}$, $125 \mu \mathrm{M}, 250 \mu \mathrm{M}$ and $500 \mu \mathrm{M}$ or $200 \mu \mathrm{M} \mathrm{H}_{2} \mathrm{O}_{2}$. After 1 $\mathrm{h}$, annexin V green reagent (IncuCyte by Sartorius, Göttingen, Germany) was added in a final dilution of 1:400. The plate was transferred into the IncuCyte ${ }^{\circledR}$ S3 Live-Cell Analysis System (Sartorius, Göttingen, Germany) and after pre-warming, the first image was recorded about $1.5 \mathrm{~h}$ after exposure. Images were recorded every $2 \mathrm{~h}$ up to $276 \mathrm{~h}$ and the experiment was performed with 3 biological replicates per group and three times independently. Confluence and apoptotic cells were determined by the IncuCyte ${ }^{\circledR} \mathrm{S} 3$ Software (Sartorius, Göttingen, Germany).

\section{Cell morphology by HE staining}

20,000 cells were plated onto coverslips in 4-well plates and grown overnight. After washing once with PBS, cells were fixed with $4 \%$ PFA in PBS for $30 \mathrm{~min}$ at $4{ }^{\circ} \mathrm{C}$. Slips were washed three times with PBS, once with ultra-pure water 
and stained with $500 \mu \mathrm{L}$ Mayer's hematoxylin (Bio-Optica, Milano, Italy) for $5 \mathrm{~min}$ at room temperature. To develop the staining, all wells were washed three times with $500 \mu \mathrm{L}$ tap water followed by once with ultra-pure water. Eosin Y $1 \%$ aqueous solution (Bio-Optica, Milano, Italy) was added with $500 \mu \mathrm{L}$ per well and stained for $3.5 \mathrm{~min}$ at room temperature. After washing twice with ultra-pure water, stained cells were dehydrated by dipping the coverslips three times into $90 \%$ EtOH and 12 times into xylenes (Sigma-Aldrich, St. Louis, USA) and mounted onto microscopy slides (Thermo Fisher Scientific, Waltham, USA) using Entellan Neu mounting medium (Merck, Darmstadt, Germany).

\section{Fluorogenic SA- $\beta$-gal staining and flow cytometric analysis}

For fluorogenic staining, 100,000 senescent or non-senescent cells were plated per well into a 24 -well plate and grown overnight. Staining was performed with the quantitative cellular senescence assay kit (Cell Biolabs, Inc., San Diego, USA) according to manufacturer's protocol. Briefly, culture medium was removed, $2 \mathrm{~mL}$ of $1 \mathrm{x}$ cell pretreatment solution (1:1000 dilution of 1000x stock in culture medium) was added and incubated for $2 \mathrm{~h}$. After adding $10 \mu \mathrm{L}$ of $200 \mathrm{x}$ SA- $\beta$-gal substrate solution directly to the cells, incubation was performed overnight. After washing three times with $3 \mathrm{~mL}$ PBS, one image was recorded using the IncuCyte ${ }^{\circledR}$ system and afterwards, cells were detached, resuspended in PBS $+2 \%$ FBS and 10,000 cells each analyzed with the ImageStreamX MarkII instrument (Luminex Corporation, Austin, USA). Software used for data acquisition was ISX and for data analysis IDEAS (both Luminex Corporation, Austin, USA). The experiment was performed three times independently.

\section{Differences in gene expression via qRT-PCR}

Non-senescent and $40 \mu \mathrm{M}$ SM-induced senescent cells each from three individual donor materials were re-plated and 3 days later (i.e., 21 day post-exposure) detached by cell scraper (Greiner AG, Kremsmünster, Austria) directly into the culture medium. For cytokine gene expression analysis, $40 \mu \mathrm{M} \mathrm{SM}$ and $200 \mu \mathrm{M} \mathrm{H}_{2} \mathrm{O}_{2}$-induced senescent cells were additionally prepared. Cells were pelleted and total RNA was directly extracted according to manufacturer's protocol using RNeasy Mini Kit (Qiagen, Hilden, Germany). Briefly, the cell pellet was disrupted by adding $350 \mu \mathrm{L}$ of buffer RLT, vortexed for $1 \mathrm{~min}$, lysate was transferred into a QIAshredder spin column (Qiagen, Hilden, Germany) and centrifuged $2 \mathrm{~min}$ at full speed. $350 \mu \mathrm{L}$ of $70 \% \mathrm{EtOH}$ was added to the homogenized lysate and mixed by pipetting. The mixture was added to a spin column and centrifuged for $30 \mathrm{~s}$ at full speed. The column was washed three times, first with $700 \mu \mathrm{L}$ buffer RW1 (30 s) and second (30 s) and third time (2 min) with $500 \mu \mathrm{L}$ buffer RPE each. Spin column was centrifuged dry for $1 \mathrm{~min}$ at full speed. RNA was eluted twice with $20 \mu \mathrm{L}$ nuclease free water (Qiagen, Hilden, Germany) for $1 \mathrm{~min}$ at full speed into a biopure $1.5 \mathrm{~mL}$ tube (Eppendorf AG, Hamburg, Germany) and stored at $-80^{\circ} \mathrm{C}$.

RNA concentration was determined by NanoQuant with plate reader infinite M200 Pro (Tecan Group AG, Männedorf, Switzerland). For each qRT-PCR plate, 500 ng RNA were separately reverse transcribed into cDNA using $\mathrm{RT}^{2}$ First Strand Kit (Qiagen, Hilden, Germany) according to manufacturer's protocol. Briefly, genomic DNA was eliminated with $2 \mu \mathrm{L}$ buffer GE, RNA and total volume of $10 \mu \mathrm{L}$ was obtained with nuclease free water in $0.2 \mathrm{~mL}$ PCR-tubes (Eppendorf AG, Hamburg, Germany). Mixture was incubated for 5 min at $42{ }^{\circ} \mathrm{C}$ and then immediately incubated on ice for at least $1 \mathrm{~min}$. The reverse transcription mix was prepared by mixing $4 \mu \mathrm{L} 5 \mathrm{x}$ Buffer BC3, $1 \mu \mathrm{L}$ Control P2, $2 \mu \mathrm{L}$ RE3 Reverse Transcriptase Mix and $3 \mu \mathrm{L}$ RNase-free water per sample. This mix was added to the $10 \mu \mathrm{L}$ genomic DNA elimination mix, mixed by pipetting, incubated for $15 \mathrm{~min}$ at $42{ }^{\circ} \mathrm{C}$ and reaction stopped by incubation for $5 \mathrm{~min}$ at $95^{\circ} \mathrm{C}$. All incubations were performed with the Mastercycler nexus GX2 (Eppendorf AG, Hamburg, Germany). After adding 91 $\mu \mathrm{L}$ RNase-free water to each tube, samples were stored at $-20{ }^{\circ} \mathrm{C}$ for a maximum of 1 week.

The following $\mathrm{RT}^{2}$ Profiler ${ }^{\mathrm{TM}}$ PCR Arrays were used: Human Cell Motility, Human Cellular Senescence, Human DNA Damage Signaling Pathway, Human DNA Repair, Human Wound Healing (Qiagen, Hilden, Germany). Additionally, a custom designed array was used to detect various cytokine, chemokine or growth factor gene transcripts (Qiagen, Hilden, Germany). For each plate, $102 \mu \mathrm{L}$ of one cDNA template was diluted with $1284 \mu \mathrm{L}$ nuclease free water and placed into the Freedom Evo automated pipetting system with EVOware Standard software (Tecan Group AG, Männedorf, Switzerland) together with one tube of $\mathrm{RT}^{2}$ SYBR Green ROX qPCR Mastermix (Qiagen, Hilden, Germany) and the PCR array plate. After extensive treatment with 7\% sodium hypochlorite (Carl Roth, Karlsruhe, Germany) and washing with ultra-pure water of the tips, $1350 \mu \mathrm{L}$ mastermix were added to the diluted cDNA. The mixture was briefly vortexed and for each well, $25 \mu \mathrm{L}$ of the cDNA-mastermix mixture were added. Before, in between and after the addition, tips were extensively washed with ultra-pure water. The plate was sealed and run on Eppendorf Mastercycler ${ }^{\circledR}$ epgradient $S$ realplex ${ }^{2}$ with Mastercycler ep realplex software (both Eppendorf AG, Hamburg, Germany) with initial activation of polymerase for $10 \mathrm{~min}$ at $95{ }^{\circ} \mathrm{C}$ followed by 40 cycles of $15 \mathrm{~s}$ at $95^{\circ} \mathrm{C}$ and $1 \mathrm{~min}$ at $60^{\circ} \mathrm{C}$. Before data were exported, threshold was manually set to 200 and the drift correction was activated for better 
comparison. All experiments were performed three times independently.

$\mathrm{C}_{\mathrm{T}}$ values were exported and analyzed by GeneGlobe (http://www.qiagen.com/geneglobe) using $\mathrm{C}_{\mathrm{T}}$ cut-off of 30 , RPLP0 housekeeping gene for normalization, fold regulation cut-off of 2 and $p$ value cut-off of 0.05 . Shown are the fold changes of senescent to control levels obtained.

\section{Western blot}

For western blots, senescent and non-senescent MSCs were plated at $10^{5}$ cells per well in 6 -well plates, and 2 days later cells were extracted. Cells were washed three times with PBS and $100 \mu \mathrm{L}$ extraction buffer $(6.25 \mathrm{mM}$ Tris- $\mathrm{HCl} \mathrm{pH}$ 7.5, $12.5 \mathrm{mM} \mathrm{NaCl}, 2.5 \mathrm{mM}$ EDTA, 1.5\% Triton X-100, one Complete Mini Inhibitor Cocktail [Roche, Basel, Switzerland] and one PhosSTOP [Roche, Basel, Switzerland] in ultra-pure water) were added per well and cell lysis performed for $15 \mathrm{~min}$ on ice. All further steps were performed on ice. Cells were scraped using a cell scraper (Greiner AG, Kremsmünster, Austria), cell suspension transferred into a centrifuge tube (Eppendorf AG, Hamburg, Germany), vortexed and disrupted by ultrasonic homogenizer (Bandelin electronic, Berlin, Germany) three times for 10 s, 0.3 interval and 30\% intensity. To complete cell disruption, incubation in extraction buffer was performed for $1 \mathrm{~h}$ in total with vortexing several times in between. Cell debris was eliminated by centrifugation at full speed for $10 \mathrm{~min}$ at $4{ }^{\circ} \mathrm{C}$. Cell lysates were divided in $25 \mu \mathrm{L}$ aliquots and stored at $-20^{\circ} \mathrm{C}$.

Cell lysates were denatured by adding $8 \mu \mathrm{L}$ loading buffer (60 $\mu \mathrm{L} 4 \mathrm{x}$ protein loading dye [LI-COR Biosciences, Lincoln, USA] and $40 \mu \mathrm{L} 3.12 \mathrm{mg}$ DTT [Sigma-Aldrich, St. Louis, USA] in ultra-pure water) per aliquot and incubation for $5 \mathrm{~min}$ at $95^{\circ} \mathrm{C}$. Denatured cell lysates and $3 \mu \mathrm{L}$ Cameleon $^{\text {TM }}$ Duo Pre-stained Protein Ladder (LI-COR Biosciences, Lincoln, USA) were separated on NuPAGE 4-12\% Bis-Tris gels $1.0 \mathrm{~mm} \times 10$ wells with NuPAGE MES SDS Running Buffer (both Novex by Thermo Fisher Scientific, Waltham, USA) for $60 \mathrm{~min}$ at $50 \mathrm{~mA}$ using a Mini Gel Tank (Invitrogen by Thermo Fisher Scientific, Waltham, USA). Immobilon ${ }^{\circledR}$-Fl PVDF Membrane with $0.45 \mu \mathrm{m}$ pore size (Merck, Darmstadt, Germany) was activated by rinsing with methanol (Sigma-Aldrich, St. Louis, USA) and transfer was performed with NuPAGE Transfer Buffer and $500 \mu \mathrm{L}$ NuPAGE Antioxidant (both Novex by Thermo Fisher Scientific, Waltham, USA) for $90 \mathrm{~min}$ at $100 \mathrm{~V}$ and $300 \mathrm{~mA}$ using Mini Blot Module (Invitrogen by Thermo Fisher Scientific, Waltham, USA).

All subsequent steps were performed with gentle rocking and washing three times with PBS-T $(0.1 \%$ Tween 20 in PBS) for $10 \mathrm{~min}$ each. Membranes were blocked for $30 \mathrm{~min}$ in Odyssey ${ }^{\circledR}$ PBS Blocking Buffer (LI-COR Biosciences, Lincoln, USA). Primary antibodies were diluted in antibody diluent (Odyssey Blocking buffer with $0.1 \%$ Tween20) and membranes were incubated overnight at $4{ }^{\circ} \mathrm{C}$. The following primary antibodies were used: Rabbit Anti-CDKN2A/ p16INK4a (ab81278 abcam, Cambridge, UK) 1:2000, Purified Mouse Anti-p21 (556,431 BD Pharmingen by Becton Dickinson, Franklin Lakes, USA) 1:250. After washing, secondary antibody IRDye ${ }^{\circledR} 800 \mathrm{CW}$ Goat Anti-Mouse or IRDye $^{\circledR} 800 \mathrm{CW}$ Goat Anti-Rabbit (LI-COR Biosciences, Lincoln, USA) were added 1:8000 in antibody diluent for $90 \mathrm{~min}$ at room temperature. After washing, the membrane was developed on Odyssey Clx with Image Studio software (LI-COR Biosciences, Lincoln, USA). For normalization, anti-actin (sc-8432 Santa Cruz, Santa Cruz, USA) primary antibody was used 1:5,000 in antibody diluent and secondary IRDye ${ }^{\circledR}$ 680RD Goat Anti-Mouse (LI-COR Biosciences, Lincoln, USA) as described above. Samples of four independent experiments were used.

\section{Clonogenic potential}

1000 cells of senescent and non-senescent MSCs were seeded per well in a 6-well plate and grown without medium exchange for 10 days. After washing with PBS, fixation with $4 \%$ PFA in PBS for 30 min at $4{ }^{\circ} \mathrm{C}$, again washing with PBS and twice with ultra-pure water, colonies were stained with $1 \mathrm{~mL}$ of $1 \%$ aqueous crystal violet solution (Sigma-Aldrich, St. Louis, USA) for $2 \mathrm{~min}$ at room temperature. After extensive washing with ultra-pure water, plates were air dried and pictures of colonies were taken with Nikon Eclipse TS100 microscope (Nikon, Minato, Japan). Afterwards, each well was destained with $500 \mu \mathrm{L}$ methanol for $20 \mathrm{~min}$ at room temperature and $150 \mu \mathrm{L}$ each were transferred to two wells of a 96-well plate and absorbance at $570 \mathrm{~nm}$ was read by plate reader infinite M200 Pro (Tecan Group AG, Männedorf, Switzerland). The absorbance of five independent experiments was normalized to corresponding controls.

\section{Migration analysis}

1000 senescent and non-senescent cells were seeded in their standard medium into a modified Boyden chamber with 24-well FluoroBlok ${ }^{\mathrm{TM}}$ cell culture insert system containing $8 \mu \mathrm{m}$ pores (Corning, Inc., Corning, USA) in 24-well plates (BD Falcon ${ }^{\mathrm{TM}}$ by Corning, Inc., Corning, USA). Migration was performed for $8 \mathrm{~h}$ and cells were fixed with $4 \%$ PFA in PBS for $30 \mathrm{~min}$ at $4{ }^{\circ} \mathrm{C}$. The membrane was cut, transferred to a coverslip, and mounted using mounting medium containing 4,6-diamidino-2-phenylindole (DAPI, Vectashield, Vector Laboratories, Inc., Burlingame, USA). The number of migrated cells was counted microscopically (Nikon, Minato, Japan) with three biological replicates per group and the experiment was performed three times independently. 
Migration was also assessed using the IncuCyte S3 system (Sartorius, Göttingen, Germany). Briefly, senescent and non-senescent cells were detached, washed once with PBS and resuspended in medium containing only $1 \%$ FBS. 1000 cells in $60 \mu \mathrm{L}$ were seeded per well into the IncuCyte ClearView insert plate (Sartorius, Göttingen, Germany) containing pores of $8 \mu \mathrm{m}$ diameter, and cells were left to settle at room temperature for $20 \mathrm{~min}$. Then, $200 \mu \mathrm{L}$ standard culture medium containing $20 \%$ FBS was added per well into the reservoir plate (Sartorius, Göttingen, Germany). The insert plate was put on top and imaging was started $30 \mathrm{~min}$ after placing into the instrument and then every $2 \mathrm{~h}$ for $160 \mathrm{~h}$ in total. Using the IncuCyte Chemotaxis analysis software, a mask was designed to recognize cells at the top (non-migrated) and bottom (migrated) of the insert-plate. To exclude proliferation, the covered area of the bottom was divided by the covered area of the top. The experiment was performed with 8 wells per condition and three times independently.

\section{Wound closure by scratch assay}

17,000 cells in $200 \mu \mathrm{L}$ were seeded in total per well into an ImageLock 96-well plate (Sartorius, Göttingen, Germany), left to settle at room temperature for 20-30 $\mathrm{min}$ and incubated overnight. To study different fractions of senescent cells vs. non-senescent cells, $10 \%, 25 \%$ and $50 \%$ were added together with $90 \%, 75 \%$ and $50 \%$ nonsenescent cells, respectively. The scratch was done with the IncuCyte WoundMaker (Sartorius, Göttingen, Germany) according to manufacturer's protocol. Briefly, the WoundMaker was washed in sterile ultra-pure water and sterilized in $80 \% \mathrm{EtOH}$, both for $5 \mathrm{~min}$. Meanwhile, medium was aspirated and $100 \mu \mathrm{L}$ fresh medium per well added. The plate was inserted into the WoundMaker and a uniform 700-800 $\mu \mathrm{m}$ scratch was created simultaneously in all 96 wells. The medium was aspirated, the scratched cells washed away twice with $100 \mu \mathrm{L}$ PBS per well and finally $200 \mu \mathrm{L}$ fresh medium was added. The plate was placed into the IncuCyte S3 (Sartorius, Göttingen, Germany) and first image was recorded $30 \mathrm{~min}$ later, then every $2 \mathrm{~h}$ up to $234 \mathrm{~h}$. The WoundMaker was cleaned by a series of washing steps with $0.5 \%$ Alconox $^{\circledR}$ (Sigma-Aldrich, St. Louis, USA), 1\% Virkon S (Antec International Limited, Sudbury, UK), sterile ultra-pure water and $80 \% \mathrm{EtOH}$ each for $5 \mathrm{~min}$ before storage. Using the IncuCyte Scratch Wound analysis software, a mask was designed to recognize the wounded area as well as the cell-covered surface. The percentage of the wound closure was determined with 8 wells per condition from four independent experiments.

\section{Secretome analysis by Bio-Plex}

For the generation of the samples, MSCs were seeded at $2.1 \times 10^{4}$ cells per $\mathrm{cm}^{2}$ in a 24 -well plate and allowed to attach overnight. The medium was removed and replaced with $600 \mu \mathrm{L}$ fresh medium supplemented or not with EtOH / $\mathrm{SM} / \mathrm{H}_{2} \mathrm{O}_{2}$. Exactly $24 \mathrm{~h}$ later, the supernatant was centrifuged at $4{ }^{\circ} \mathrm{C}$ and $206 \times$ g for $15 \mathrm{~min}$, divided at $140 \mu \mathrm{L}$ each in four $0.5 \mathrm{~mL}$ Protein LoBind Tubes (Eppendorf AG, Hamburg, Germany) and stored at $-80{ }^{\circ} \mathrm{C}$. Samples were taken at days 1, 7, 14, 21 and 28 after exposure.

The samples were analyzed using the Bio-Plex Pro $^{\text {TM }}$ Human Chemokine 40-plex Assay and Bio-Plex Pro ${ }^{\mathrm{TM}}$ Human Inflammation 37-plex Assay (BioRad, Hercules, USA) according to manufacturer's protocol. All incubation steps were performed, while plate was covered with provided aluminum foil at room temperature and at $850 \mathrm{rpm}$ on $\mathrm{IKA}^{\circledR}$ MS3 digital shaker (IKA, Staufen, Germany). Washing steps were performed three times with $100 \mu \mathrm{L}$ wash buffer per well using the wash station HydroFlex (Tecan Group AG, Männedorf, Swiss). Briefly, $50 \mu \mathrm{L}$ beads were added per well into the Bio-Plex ${ }^{\circledR}$ 96-well plate and washed. Standards were diluted as described in cell culture medium and $50 \mu \mathrm{L}$ were added per well as well as $50 \mu \mathrm{L}$ of the samples generated. The plate was incubated for $1 \mathrm{~h}$. After washing, $50 \mu \mathrm{L}$ detection antibodies were added per well and incubated for $30 \mathrm{~min}$. After washing, $50 \mu \mathrm{L}$ SA-PE were added per well and incubated for $10 \mathrm{~min}$. After the final washing, beads were resuspended in $125 \mu \mathrm{L}$ assay buffer by shaking for $30 \mathrm{~s}$. Plates were analyzed by the Bio-Plex ${ }^{\circledR} 200$ System and Realplex software (BioRad, Hercules, USA). The Experiment was performed in biological duplicates per group and three times independently.

\section{Secretome analysis by cytokine array}

For sample generation, 42,500 non-senescent or senescent $\left(10 \mu \mathrm{M}\right.$ SM, $40 \mu \mathrm{M}$ SM and $\left.200 \mu \mathrm{M} \mathrm{H}_{2} \mathrm{O}_{2}\right)$ MSCs were seeded per well in a 24-well plate and grown overnight. Medium was removed and replaced with $1000 \mu \mathrm{L}$ fresh medium. After exactly $24 \mathrm{~h}$, the supernatant was removed, centrifuged at $24,696 \times \mathrm{g}$ at RT for $10 \mathrm{~min}$ and the supernatant immediately used as sample.

All samples were analyzed with RayBio ${ }^{\circledR}$ Human Cytokine Antibody Array G-Series 6, 7, 8, 9 and 10 (RayBiotech, Peachtree Corners, USA) according to manufacturer's instructions. Briefly, array slides were transferred to RT and thawed for $15 \mathrm{~min}$. Arrays were opened, cover removed and dried for $2 \mathrm{~h}$ in a laminar flow hood. All incubation steps were performed at room temperature and with $850 \mathrm{rpm}$ on IKA ${ }^{\circledR}$ MS3 digital shaker (IKA, Staufen, Germany), while arrays were sealed with provided adhesive film for steps $>2 \mathrm{~h}$ or $<100 \mu \mathrm{L}$ per well. $100 \mu \mathrm{L}$ blocking solution 
(0.05\% Tween20 [Sigma-Aldrich, St. Louis, USA] in Odyssey Blocking Buffer [LI-COR Biosciences, Lincoln, USA]) were added per well and incubated for $1 \mathrm{~h}$. After removal of blocking solution, samples as described above and culture medium as blank were added with $100 \mu \mathrm{L}$ per well and incubated at $4{ }^{\circ} \mathrm{C}$ overnight. The samples were removed, and arrays washed $3 \mathrm{x}$ for $5 \mathrm{~min}$ and $150 \mu \mathrm{L}$ Wash Buffer I (Wash Buffer I concentrate 1:20 diluted in ultra-pure water) per well each. Each slide was submerged in Wash Buffer I $2 \mathrm{x}$ for $10 \mathrm{~min}$ each. The last two steps were repeated with Wash Buffer II (Wash Buffer II concentrate 1:20 diluted in ultrapure water). $70 \mu \mathrm{L}$ Biotin-conjugated anti-cytokines (stock reagent diluted with $300 \mu \mathrm{L}$ blocking solution) were added per well and incubated for $2 \mathrm{~h}$. Arrays were washed thrice with $150 \mu \mathrm{L}$ Wash Buffer I and twice with $150 \mu \mathrm{L}$ Wash Buffer II each for 5 min. IRDye ${ }^{\circledR} 800 \mathrm{CW}$ Streptavidin antibody (1:3,000 in blocking solution, LI-COR Biosciences, Lincoln, USA) was added with $70 \mu \mathrm{L}$ per Well and incubated for $45 \mathrm{~min}$. Slides were finally washed four times with 150 $\mu \mathrm{L}$ Wash Buffer I, twice with $150 \mu \mathrm{L}$ Wash Buffer II each for $5 \mathrm{~min}$ and with removed frame in provided centrifuge tube twice each with Wash Buffer I and II for 10 min each. All glass slides were scanned at $800 \mathrm{~nm}$ on Odyssey Clx with Image Studio software (LI-COR Biosciences, Lincoln, USA). The experiment was performed two times independently with biological duplicates per group.

\section{Generation of conditioned medium and assaying its influence on migration}

Conditioned medium was generated like the supernatants for the secretome analysis. Briefly, $1.2 \times 10^{6}$ senescent or non-senescent cells were seeded per T75 cell culture flask (Greiner AG, Kremsmünster, Austria). The following day, $20 \mathrm{~mL}$ fresh medium was added to each flask and $24 \mathrm{~h}$ later, the conditioned medium was transferred to a centrifuge tube (Greiner AG, Kremsmünster, Austria), centrifuged at 522×g for $5 \mathrm{~min}$ and the supernatant filtered with $0.2 \mu \mathrm{m}$ syringe filters (VWR International, Radnor, USA) on SOFT-JECT ${ }^{\circledR}$ syringes (Henke-Sass Wolf, Tuttlingen, Germany). The filtered conditioned medium was divided in aliquots and stored at $-80{ }^{\circ} \mathrm{C}$.

To assess the influence of conditioned medium on the migration of healthy MSCs, the IncuCyte migration tool was used as described above. Therefore, only untreated low-passage MSCs were seeded in the insert plate and regular cell culture medium or conditioned medium was added to the reservoir plate. To reduce the potential problem of nutrient deficiency, also a mixture of $50 \%$ conditioned medium with $50 \%$ cell culture medium was used. The plates were recorded every $2 \mathrm{~h}$ for $188 \mathrm{~h}$ in total. The experiment was performed with 8 wells per condition and three times independently.

\section{Selective removal by senolytics}

Non-senescent and senescent cells were seeded at 6,735 cells per well in a 96-well plate (Greiner AG, Kremsmünster, Austria) including medium controls each for background determination. The following day, the cells were exposed to increasing concentrations of senolytic drugs shown in Table S2 as well as solvent controls for $5 \mathrm{~d}$ or $24 \mathrm{~h}$. Then cells were washed once with PBS and XTT staining solution (Sigma-Aldrich, St. Louis, USA) was prepared by mixing $5 \mathrm{~mL}$ XTT labeling reagent with $100 \mu \mathrm{L}$ electron-coupling reagent per plate. Cells were incubated with $100 \mu \mathrm{L}$ medium and $50 \mu \mathrm{L}$ XTT staining solution and absorbance was determined at $450 \mathrm{~nm}$ with the reference set to $630 \mathrm{~nm}$. Background absorbance was removed using wells only containing medium and viability was normalized to solvent controls. All experiments were performed with 4 biological replicates per group and three times (except 17-DMAG and dasatinib $24 \mathrm{~h}$ each two times) independently.

\section{Statistical analysis}

Since the cells were isolated from different donors, differences between independent experiments somehow reflect variations derived from age, sex, and other parameters, which more closely resembles the biological nature than the use of immortalized cell lines or pools of donor materials. Overall, the results were still comparable between donors as previous studies showed (Schmidt et al. 2013).

Each experiment was repeated as indicated. The statistic software RStudio (version 1.2.1335 with $\mathrm{R}$ version 3.6.1) was used for statistical analysis and graphic presentation (RStudio Inc., Boston, USA). For viability tests, non-linear regression was performed and displayed as mean with $99 \%$ confidence interval. Means and smoothing function with 99\% confidence intervals are displayed for representation of SA- $\beta$-gal and IncuCyte results. Otherwise, Tukey boxplots and single data points are shown. qPCR results are shown as means only for fold regulations $>2.0$ or $<-2.0$ and $p<0.05$. Senescent cells were compared to controls by the unpaired two-samples Wilcoxon test. $p$ values $<0.05$ were considered statistically significant.

\section{Availability of data and material}

All data generated or analyzed during this study are included in this published article and its supplementary information.

Acknowledgements The authors like to thank Prof. Dr. Harry Scherthan (Bundeswehr Institute of Radiobiology) and his group for the irradiation of the cells and Emine Cukur, Ram Prasad and Cornelia Muschik for their excellent technical support. This work was financed by the German Ministry of Defense. 
Author contributions Conceptualization, AS, SR, AB, and AM; Methodology, AS and SR; Investigation, SR, NJ, NM, TM and AN; Resources, MS, CR; Supervision, AS, AB, DS and HT; Writing, SR; and Funding Acquisition, HT.

Funding Open Access funding enabled and organized by Projekt DEAL. This study was funded by the German Ministry of Defense.

\section{Compliance with ethical standards}

Conflicts of interest The authors declare that they have no conflict of interest.

Ethical approval and informed consent Human MSCs were isolated from bone marrow of femoral heads, which were obtained with informed consent from the HELIOS Amper Hospital in Dachau, Germany, or Wolfart Hospital in Gräfelfing, Germany. All experiments with human cell material was performed according to authorization of the ethics committee of Ludwig Maximilian University Munich, Germany.

Open Access This article is licensed under a Creative Commons Attribution 4.0 International License, which permits use, sharing, adaptation, distribution and reproduction in any medium or format, as long as you give appropriate credit to the original author(s) and the source, provide a link to the Creative Commons licence, and indicate if changes were made. The images or other third party material in this article are included in the article's Creative Commons licence, unless indicated otherwise in a credit line to the material. If material is not included in the article's Creative Commons licence and your intended use is not permitted by statutory regulation or exceeds the permitted use, you will need to obtain permission directly from the copyright holder. To view a copy of this licence, visit http://creativecommons.org/licenses/by/4.0/.

\section{References}

Abreu-Blanco MT, Watts JJ, Verboon JM, Parkhurst SM (2012) Cytoskeleton responses in wound repair. Cell Mol Life Sci 69:2469-2483

Alessio N, Del Gaudio S, Capasso S et al (2015) Low dose radiation induced senescence of human mesenchymal stromal cells and impaired the autophagy process. Oncotarget 6:8155-8166. https ://doi.org/10.18632/oncotarget.2692

Augustin M, Maier K (2003) Psychosomatic aspects of chronic wounds. Dermatol Psychosom/Dermatol Psychosom 4:5-13. https ://doi.org/10.1159/000070529

Baar MP, Brandt RMC, Putavet DA et al (2017) Targeted apoptosis of senescent cells restores tissue homeostasis in response to chemotoxicity and aging. Cell 169:132-147.e16. https://doi. org/10.1016/j.cell.2017.02.031

Baker DJ, Wijshake T, Tchkonia T et al (2011) Clearance of p16Ink4apositive senescent cells delays ageing-associated disorders. Nature 479:232-236. https://doi.org/10.1038/nature10600

Baker DJ, Childs BG, Durik M et al (2016) Naturally occurring p16Ink4a-positive cells shorten healthy lifespan. Nature 530:184 189. https://doi.org/10.1038/nature16932

Behboudi H, Noureini SK, Ghazanfari T, Ardestani SK (2018) DNA damage and telomere length shortening in the peripheral blood leukocytes of 20 years SM-exposed veterans. Int Immunopharmacol 61:37-44. https://doi.org/10.1016/j.intimp.2018.05.008

Behravan E, Moallem SA, Kalalinia F et al (2018) Telomere shortening associated with increased levels of oxidative stress in sulfur mustard-exposed Iranian veterans. Mutat Res Toxicol Environ Mutagen 834:1-5. https://doi.org/10.1016/j.mrgen tox.2018.06.017

Borodkina AV, Deryabin PI, Giukova AA, Nikolsky NN (2018) "Social Life" of Senescent Cells: What Is SASP and Why Study It? Acta Naturae 10:4-14

Brandl A, Meyer M, Bechmann V et al (2011) Oxidative stress induces senescence in human mesenchymal stem cells. Exp Cell Res 317:1541-1547. https://doi.org/10.1016/j.yexcr.2011.02.015

Brimfield AA, Soni SD, Trimmer KA et al (2012) Metabolic activation of sulfur mustard leads to oxygen free radical formation. Free Radic Biol Med 52:811-817. https://doi.org/10.1016/j.freeradbio med.2011.11.031

Bruder SP, Kurth AA, Shea M et al (1998) Bone regeneration by implantation of purified, culture-expanded human mesenchymal stem cells. J Orthop Res 16:155-162. https://doi.org/10.1002/ jor.1100160202

Bukowiecki A, Hos D, Cursiefen C, Eming S (2017) Wound-healing studies in cornea and skin: parallels, differences and opportunities. Int J Mol Sci 18:1257. https://doi.org/10.3390/ijms18061257

Byun HO, Lee YK, Kim JM, Yoon G (2015) From cell senescence to age-related diseases: differential mechanisms of action of senescence-associated secretory phenotypes. BMB Rep 48:549-558

Carlos Sepúlveda J, Tomé M, Eugenia Fernández M et al (2014) Cell senescence abrogates the therapeutic potential of human mesenchymal stem cells in the lethal endotoxemia model. Stem Cells 32:1865-1877. https://doi.org/10.1002/stem.1654

Chang J, Wang Y, Shao L et al (2016) Clearance of senescent cells by ABT263 rejuvenates aged hematopoietic stem cells in mice. Nat Med 22:78-83. https://doi.org/10.1038/nm.4010

Collado M, Blasco MA, Serrano M (2007) Cellular senescence in cancer and aging. Cell 130:223-233. https://doi.org/10.1016/j. cell.2007.07.003

Coppé J-P, Patil CK, Rodier F et al (2008) Senescence-associated secretory phenotypes reveal cell-nonautonomous functions of oncogenic RAS and the p53 tumor suppressor. PLoS Biol 6:28532868. https://doi.org/10.1371/journal.pbio.0060301

Cullumbine H (1947) Medical aspects of mustard gas poisoning Nature 159:151-153. https://doi.org/10.1038/159151a0

Demaria M, Ohtani N, Youssef SA et al (2014) An essential role for senescent cells in optimal wound healing through secretion of PDGF-AA. Dev Cell 31:722-733. https://doi.org/10.1016/j.devce 1.2014.11.012

Dörr JR, Yu Y, Milanovic M et al (2013) Synthetic lethal metabolic targeting of cellular senescence in cancer therapy. Nature 501:421425. https://doi.org/10.1038/nature12437

Emadi SN, Mortazavi M, Mortazavi H (2008) Late cutaneous manifestations 14 to 20 years after wartime exposure to sulfur mustard gas: a long-term investigation. Arch Dermatol 144:1059-1061. https://doi.org/10.1001/archderm.144.8.1059

Etemad L, Moshiri M, Balali-Mood M (2019) Advances in treatment of acute sulfur mustard poisoning - a critical review. Crit Rev Toxicol 49:191-214. https://doi.org/10.1080/10408444.2019.1579779

Fuhrmann-Stroissnigg H, Ling YY, Zhao J et al (2017) Identification of HSP90 inhibitors as a novel class of senolytics. Nat Commun 8:422. https://doi.org/10.1038/s41467-017-00314-z

Grezella C, Fernandez-Rebollo E, Franzen J et al (2018) Effects of senolytic drugs on human mesenchymal stromal cells. Stem Cell Res Ther 9:108. https://doi.org/10.1186/s13287-018-0857-6

Hassan ZM, Ebtekar M (2002) Immunological consequence of sulfur mustard exposure. Immunol Lett 83:151-152. https://doi. org/10.1016/S0165-2478(02)00076-7

Janssens R, Struyf S, Proost P (2018) The unique structural and functional features of CXCL12. Cell Mol Immunol 15:299-311

John H, Koller M, Worek F et al (2019) Forensic evidence of sulfur mustard exposure in real cases of human poisoning by detection of 
diverse albumin-derived protein adducts. Arch Toxicol 93:18811891. https://doi.org/10.1007/s00204-019-02461-2

Jun J-I, Lau LF (2010) The matricellular protein CCN1 induces fibroblast senescence and restricts fibrosis in cutaneous wound healing. Nat Cell Biol 12:676-685. https://doi.org/10.1038/ncb2070

Kearney AY, Anchang B, Plevritis S, Felsher DW (2015) ARF: connecting senescence and innate immunity for clearance. Aging (Albany NY) 7:613-615. https://doi.org/10.18632/aging.100813

Kilic E, Ortatatli M, Sezigen S et al (2018) Acute intensive care unit management of mustard gas victims: the Turkish experience. Cutan Ocul Toxicol 37:332-337. https://doi.org/10.1080/15569 527.2018.1464018

Kim YH, Choi YW, Lee J et al (2017) Senescent tumor cells lead the collective invasion in thyroid cancer. Nat Commun 8:15208. https ://doi.org/10.1038/ncomms15208

Krizhanovsky V, Yon M, Dickins RA et al (2008) Senescence of activated stellate cells limits liver fibrosis. Cell 134:657-667. https:// doi.org/10.1016/j.cell.2008.06.049

Lee DE, Ayoub N, Agrawal DK (2016) Mesenchymal stem cells and cutaneous wound healing: novel methods to increase cell delivery and therapeutic efficacy. Stem Cell Res Ther 7:37. https://doi. org/10.1186/s13287-016-0303-6

Ma S, Xie N, Li W et al (2014) Immunobiology of mesenchymal stem cells. Cell Death Differ 21:216-225. https://doi.org/10.1038/ cdd. 2013.158

Malaquin N, Martinez A, Rodier F (2016) Keeping the senescence secretome under control: molecular reins on the senescence-associated secretory phenotype. Exp Gerontol 82:39-49. https://doi. org/10.1016/j.exger.2016.05.010

Minieri V, Saviozzi S, Gambarotta G et al (2015) Persistent DNA damage-induced premature senescence alters the functional features of human bone marrow mesenchymal stem cells. J Cell Mol Med 19:734-743. https://doi.org/10.1111/jcmm.12387

Nelson G, Wordsworth J, Wang C et al (2012) A senescent cell bystander effect: senescence-induced senescence. Aging Cell 11:345-349. https://doi.org/10.1111/j.1474-9726.2012.00795.x

Nie C, Yang D, Xu J et al (2011) Locally administered adiposederived stem cells accelerate wound healing through differentiation and vasculogenesis. Cell Transplant 20:205-216. https://doi. org/10.3727/096368910X520065

Ranganath SH, Levy O, Inamdar MS, Karp JM (2012) Harnessing the mesenchymal stem cell secretome for the treatment of cardiovascular disease. Cell Stem Cell 10:244-258. https://doi. org/10.1016/j.stem.2012.02.005

Rodriguez-Menocal L, Salgado M, Ford D, Van Badiavas E (2012) Stimulation of skin and wound fibroblast migration by mesenchymal stem cells derived from normal donors and chronic wound patients. Stem Cells Transl Med 1:221-229. https://doi. org/10.5966/sctm.2011-0029

Rodriguez-Menocal L, Shareef S, Salgado M et al (2015) Role of whole bone marrow, whole bone marrow cultured cells, and mesenchymal stem cells in chronic wound healing. Stem Cell Res Ther 6:24. https://doi.org/10.1186/s13287-015-0001-9

Schmidt A, Scherer M, Thiermann H, Steinritz D (2013) Mesenchymal stem cells are highly resistant to sulfur mustard. Chem Biol Interact 206:505-511. https://doi.org/10.1016/j.cbi.2013.07.013

Schmidt A, Steinritz D, Rothmiller S et al (2018a) Effects of sulfur mustard on mesenchymal stem cells. Toxicol Lett. https://doi. org/10.1016/j.toxlet.2017.08.008

Schmidt A, Steinritz D, Rudolf K-D et al (2018b) Accidental sulfur mustard exposure: a case report. Toxicol Lett 293:62-66. https:// doi.org/10.1016/j.toxlet.2017.11.023

Schreier C, Rothmiller S, Scherer MA et al (2018) Mobilization of human mesenchymal stem cells through different cytokines and growth factors after their immobilization by sulfur mustard. Toxicol Lett. https://doi.org/10.1016/j.toxlet.2018.02.011
Sedelnikova OA, Horikawa I, Zimonjic DB et al (2004) Senescing human cells and ageing mice accumulate DNA lesions with unrepairable double-strand breaks. Nat Cell Biol 6:168-170. https:// doi.org/10.1038/ncb1095

Severino V, Alessio N, Farina A et al (2013) Insulin-like growth factor binding proteins 4 and 7 released by senescent cells promote premature senescence in mesenchymal stem cells. Cell Death Dis 4:e911. https://doi.org/10.1038/cddis.2013.445

Sezigen S, Ivelik K, Ortatatli M et al (2019) Victims of chemical terrorism, a family of four who were exposed to sulfur mustard. Toxicol Lett 303:9-15. https://doi.org/10.1016/j.toxlet.2018.12.006

Sharpless NE, Sherr CJ (2015) Forging a signature of in vivo senescence. Nat Rev Cancer 15:397-408. https://doi.org/10.1038/nrc3960

Smith WJ, Sanders KM, Ruddle SE, Gross CL (1993) Cytometric analysis of DNA changes induced by sulfur mustard. Cutan Ocul Toxicol 12:337-347. https://doi.org/10.3109/15569529309050150

Sulzberger MB, Baer RL, Kanof A, Lowenberg C (1947) Skin sensitization to vesicant agents of chemical warfare. J Invest Dermatol 8:365-393

Tchkonia T, Zhu Y, van Deursen J et al (2013) Cellular senescence and the senescent secretory phenotype: therapeutic opportunities. J Clin Invest 123:966-972. https://doi.org/10.1172/JCI64098

Thiruvoth F, Mohapatra D, Sivakumar D et al (2015) Current concepts in the physiology of adult wound healing. Plast Aesthetic Res 2:250. https://doi.org/10.4103/2347-9264.158851

van Deursen JM (2014) The role of senescent cells in ageing. Nature 509:439-446. https://doi.org/10.1038/nature13193

von Zglinicki T (2002) Oxidative stress shortens telomeres. Trends Biochem Sci 27:339-344. https://doi.org/10.1016/s0968 -0004(02)02110-2

Walter MNM, Wright KT, Fuller HR et al (2010) Mesenchymal stem cell-conditioned medium accelerates skin wound healing: an in vitro study of fibroblast and keratinocyte scratch assays. Exp Cell Res 316:1271-1281. https://doi.org/10.1016/j.yexcr .2010 .02 .026

Wang KX, Denhardt DT (2008) Osteopontin: Role in immune regulation and stress responses. Cytokine Growth Factor Rev 19:333-345

Wang Y, Chen X, Cao W, Shi Y (2014) Plasticity of mesenchymal stem cells in immunomodulation: pathological and therapeutic implications. Nat Immunol 15:1009-1016. https://doi.org/10.1038/ ni. 3002

Wilkinson HN, Hardman MJ (2020) Senescence in wound repair: emerging strategies to target chronic healing wounds. Front Cell Dev Biol 8:773

Willyard C (2018) Unlocking the secrets of scar-free skin healing. Nature 563:S86-S88. https://doi.org/10.1038/d41586-018-07430-w

Yosef R, Pilpel N, Tokarsky-Amiel R et al (2016) Directed elimination of senescent cells by inhibition of BCL-W and BCL-XL. Nat Commun 7:11190. https://doi.org/10.1038/ncomms11190

Yue L, Zhang Y, Chen J et al (2015) Distribution of DNA adducts and corresponding tissue damage of sprague-dawley rats with percutaneous exposure to sulfur mustard. Chem Res Toxicol 28:532-540. https://doi.org/10.1021/tx5004886

Zhu Y, Tchkonia T, Pirtskhalava T et al (2015) The Achilles' heel of senescent cells: from transcriptome to senolytic drugs. Aging Cell 14:644-658. https://doi.org/10.1111/acel.12344

Zubel T, Hochgesand S, John H et al (2019) A mass spectrometric platform for the quantitation of sulfur mustard-induced nucleic acid adducts as mechanistically relevant biomarkers of exposure. Arch Toxicol 93:61-79. https://doi.org/10.1007/s00204-018-2324-7

Publisher's Note Springer Nature remains neutral with regard to jurisdictional claims in published maps and institutional affiliations. 